\title{
Contribution of Thrombospondin-1 and -2 to Lipopolysaccharide- Induced Acute Respiratory Distress Syndrome
}

\author{
Qiang Li, ${ }^{1}$ Xiaoxiao Fu, ${ }^{2}$ Jiang Yuan $\mathbb{D}^{3},{ }^{3}$ and Shu Han $\mathbb{D}^{2}$ \\ ${ }^{1}$ The Emergency Department, The Second Affiliated Hospital Zhejiang University School of Medicine (SAHZU), Hangzhou, China \\ ${ }^{2}$ Institute of Anatomy and Cell Biology, Medical College, Zhejiang University, Hangzhou, China \\ ${ }^{3}$ Department of Pulmonology, The Children's Hospital, Zhejiang University School of Medicine, National Clinical Research Center for \\ Child Health, Hangzhou, China
}

Correspondence should be addressed to Jiang Yuan; yuanj212@zju.edu.cn and Shu Han; han00shu@zju.edu.cn

Received 23 September 2020; Revised 12 March 2021; Accepted 30 March 2021; Published 17 April 2021

Academic Editor: Sandra Helena Penha Oliveira

Copyright ( 2021 Qiang Li et al. This is an open access article distributed under the Creative Commons Attribution License, which permits unrestricted use, distribution, and reproduction in any medium, provided the original work is properly cited.

\begin{abstract}
Thrombospondin (TSP) proteins have been shown to impact T-cell adhesion, migration, differentiation, and apoptosis. Thrombospondin-1 (TSP-1) is specifically upregulated in several inflammatory diseases and can effectively promote lipopolysaccharide- (LPS-) induced inflammation. In contrast, thrombospondin-2 (TSP-2) has been associated with activation of "anti-inflammatory" T-regulatory cells (Tregs). In this study, we investigated the effects of both TSP-1 and TSP-2 overexpression on macrophage polarization and activation in vitro and in vivo. We analyzed the effects of TSP-1 and TSP-2 on inflammation, vascular endothelial permeability, edema, ultrastructural morphology, and apoptosis in lung tissues of an ARDS mouse model and cultured macrophages. Our results demonstrated that TSP-2 overexpression effectively attenuated LPSinduced ARDS in vivo and promoted M2 macrophage phenotype polarization in vitro. Furthermore, TSP-2 played a role in regulating pulmonary vascular barrier leakage by activating the PI3K/Akt pathway. Overall, our findings indicate that TSP-2 can modulate inflammation and could therefore be a potential therapeutic target against LPS-induced ARDS.
\end{abstract}

\section{Introduction}

Acute respiratory distress syndrome (ARDS) is a diffuse alveolar injury caused by lung infection or systemic inflammation/sepsis in multiple patients. Lung inflammation can disrupt alveolar endothelial and epithelial barriers and in turn enhance the influx of multiple cell types, including neutrophils, macrophages, and other inflammatory cells that subsequently release various inflammatory and cytotoxic mediators [1]. Earlier studies reported that macrophages protect against ARDS by modulating inflammation and initiating lung repair $[2,3]$. However, it is important to highlight that the role of macrophages in regulating inflammation is dependent on the relative abundance of their specific M1 and M2 phenotypes. M1polarized macrophages are typically generated in response to microbial products or interferon- (IFN-) $\gamma$, and can efficiently produce proinflammatory cytokines, including interleukin(IL-) 6, IL-12, and tumor necrosis factor- (TNF-) $\alpha$. These cytokines induce a polarized type I immune response that inhibits cell proliferation and induces tissue damage. In contrast, M2polarized macrophages induce anti-inflammatory cytokines and promote tissue remodeling and wound healing [4].

Thrombospondins (TSPs) are nonstructural glycoproteins belonging to a family of matricellular proteins involved in regulating cell-matrix interactions. TSPs have been shown to influence T-cell adhesion, migration, differentiation, and apoptosis [5]. Among the various TSPs, thrombospondin-1 (TSP-1), which is upregulated in several inflammatory diseases [6], can effectively augment lipopolysaccharide- (LPS-) induced inflammation in THP-1 cells. Interestingly, TSP-1 ablation by siRNA has been observed to reduce IL-6, IL-1 $\beta$, and TNF- $\alpha$ secretion in THP-1 cells, while its overexpression upregulated these cytokines by regulating the NF- $\kappa \mathrm{B}$ signaling pathway [7]. The CD47 protein has been shown to be involved in TSP-1-mediated regulation of IL-1 $\beta$ [8]. Thus, TSP-1 and CD47 could be potential targets for limiting the proinflammatory effects of LPS. In contrast, thrombospondin-2 (TSP-2) has been implicated in endogenous regulation of angiogenesis 
and autoimmune inflammation and appears to be part of the protective mechanism for preventing inflammation [9]. TSP2 has also been shown to activate anti-inflammatory $\mathrm{T}$ regulatory cells (Tregs) [5]. Importantly, previous studies have also revealed that TSP-2 ablation significantly decreased the expression of phosphorylated- (p-) Akt and p-phosphatidyl inositol-3 kinase (PI3K), thereby indicating its role in PI3K/Akt pathway activation [10].

Despite information about the pro- and anti-inflammatory roles of TSP-1 and TSP-2, their relationship with M1/M2 macrophages is still unclear. Moreover, their potential role in the pathological process of ARDS requires further investigation. Therefore, in our current study, we investigated the effects of both TSP-1 and TSP-2 overexpression on macrophages in vitro and in vivo using a well-established LPS-induced ARDS mouse model. We also investigated the role of TSP-1 and TSP2 on in vitro macrophage polarization and their potential to regulate the PI3K/Akt signaling pathway.

\section{Materials and Methods}

\subsection{In Vitro Experiments}

2.1.1. Cell Culture, Transfection, and In Vitro LPS Stimulation. The MH-S murine alveolar macrophage cell line purchased from American Type Culture Collection (Manassas, VA) was initially cultured in RPMI 1640 medium containing 10\% heat-inactivated fetal calf serum (FCS) (Hyclone Laboratories, Logan, UT). Later, these cells were plated on 6-well tissue culture plates at a concentration of $5 \times 10^{5}$ cells $/ \mathrm{ml}$ in $5 \%$ $\mathrm{CO}_{2}$ at $37^{\circ} \mathrm{C}$. Cell monolayers were washed with Hank's buffered saline solution (HBSS) and replenished with $10 \%$ FCS-RPMI 1640 media without antibiotics for subsequent transfection, as described previously [11]. Full-length human TSP- 1 and TSP-2 cDNA constructs subcloned into the pEGF expression vector were transfected into the MH-S cells. The empty vector was transfected into $\mathrm{MH}-\mathrm{S}$ cells as a control. These $p E G F P-T S P 1$ and $p E G F P-T S P 2 \mathrm{cDNAs}$ were mixed with Lipofectamine PlusTM Reagent (Life Technologies) in serum and antibiotic-free media for $15 \mathrm{~min}$ at room temperature and then added to the plated cells. Forty-eight hours later, visible green fluorescence from the $\mathrm{pEGF}$ expression vector was detected in the transfected cells using fluorescence microscopy, and TSP-1 or TSP-2 protein expression was detected using Western blot analysis. For in vitro stimulation, $\mathrm{MH}-\mathrm{S}$ cells overexpressing TSP-1 and TSP-2 were first serum starved for $24 \mathrm{~h}$ and then treated with LPS $(\mathrm{L}, 10 \mu \mathrm{g} / \mathrm{ml})$ for $30 \mathrm{~min}$. $\mathrm{MH}-\mathrm{S}$ cells expressing empty vector were treated with LPS as "vehicle control" or with culture medium as "normal control."

2.1.2. Immunofluorescence Staining. After fixing cultured cells with $4 \%$ paraformaldehyde for $30 \mathrm{~min}$, the cells were washed with phosphate-buffered saline (PBS) and later incubated with primary antibodies against polyclonal rabbit anti-TSP-1 and TSP-2 (1:100; Chemicon, Euromedex, Souffelweyersheim, France), anti-CD38 (1:100; Santa Cruz Biotechnology), antiAkt and p-Akt (1:500; R\&D Systems, Minneapolis, MN, USA), anti-arginase-1 (Arg-1, 1:500; R\&D Systems, Minneapolis, MN, USA), anti-formyl peptide receptor 2 (Fpr-2, 1:500;
R\&D Systems, Minneapolis, MN, USA), anti-active caspase-3 (1:100; Santa Cruz Biotechnology, Santa Cruz, CA), and anti-early growth response protein 2 (EGR2, 1:200; Cayman Chemical, Ann Arbor, MI, USA), as described previously [12]. Omitting primary antibody controls was used to confirm the specificity of staining signals. Five areas from each slide were randomly selected for image acquisition under 200x magnification. The red immunoreactive signal indicated expression of each specific protein, while the green signal indicated TSP-1 and TSP-2 expression; the Hoechst 33342 blue signal indicated nuclei staining.

2.1.3. Enzyme-Linked Immunosorbent Assay (ELISA). The cultured supernatants from each group of cells were used to assess TNF- $\alpha$, IL- 6 , and IL- $1 \beta$ concentrations using an ELISA kit according to the manufacturer's instructions (BioLegend, Inc., USA).

\subsection{In Vivo Experiments}

2.2.1. Experimental Design. A total of 320 C57/BL6 mice were randomly divided into four groups. Three groups (100 mice per group) were injected via the tail vein with cDNA of TSP-1, TSP-2, or vehicle (empty vector), while the fourth group (20 mice) served as the normal group. The Entranster $^{\mathrm{TM}}$-in vivo kit (Engreen Biosystem Ltd., Beijing, China) was used to transfect macrophages in the host in vivo to overexpress TSP-1 and TSP-2. After mixing the cDNA of TSP-1, TSP-2, or the empty vector with the Entranster kit solutions, the mixture was injected directly into the tail veins of the mice. ARDS was induced by intravenous injection of LPS (4 mg/kg) (L4391, Sigma-Aldrich) under anesthesia with intraperitoneal sodium pentobarbital $(30 \mathrm{mg} / \mathrm{kg})$ in the TSP-1, TSP-2, and vehicle-transfected mice. To specifically observe the effects of the PI3K/Akt pathway on lung inflammation, we injected TSP-1- and TSP-2-transfected mice with LY294002 $(0.3 \mathrm{mg} / \mathrm{kg})$ via the tail vein. All animal experiments were performed according to the protocols and policies described in the Care and Use of Laboratory Animals issued by the National Institutes of Health and approved by the animal ethics committee of Zhejiang University.

\subsubsection{Histopathological Examination and Immunohistochemical} (IHC) Staining. Five mice from each of the TSP-1 cDNA, TSP-2 cDNA, and vehicle groups were sacrificed after 4, 12, 24, and $72 \mathrm{~h}$ of LPS injection. To perform histopathological examination, the animals were anaesthetized using ketamine-xylazine (120 mg/kg ketamine hydrochloride (5\%) and $1 \mathrm{mg} / \mathrm{kg}$ xylazine $(2 \%))$, and the trachea of each mouse was cannulated with an indwelling needle and later perfused with saline. In situ fixation was achieved by instillation of $4 \%$ paraformaldehyde in PBS. Subsequently, half of the lungs were incised and fixed in $4 \%$ paraformaldehyde for $4 \mathrm{~h}$ followed by equilibrium with $30 \%$ sucrose solution in PBS, while the remaining half was fixed in $10 \%$ glutaraldehyde solution for transmission electron microscopy imaging. Frozen sections $(20 \mu \mathrm{m}$ thick) were prepared using a Leica cryostat and mounted on glass slides coated with $0.02 \%$ poly-L-lysine for histological analysis and immunofluorescent staining. 
Lung histology of hematoxylin and eosin- (H\&E-) stained slides was assessed under a light microscope by an experienced pathologist blinded to the experimental conditions. The degree of lung injury was assessed by two independent pathologists and scored as follows: 0 represented no injury; 1 represented subpleural edema/fibrin and hemorrhage; 2 represented interlobular edema/fibrin and hemorrhage; 3 represented alveolar edema/fibrin and hemorrhage; 4 represented congestion of alveolar septa; and 5 represented hyaline membrane changes of the alveolar septa. The overall score from each mouse was averaged within a group to obtain an average score, and comparisons were then made between different groups $[13,14]$.

IHC staining of the frozen lung sections was performed using the following primary antibodies: rabbit anti-TSP-1 and TSP-2 (1:500, Chemicon, Euromedex, Souffelweyersheim, France), anti-Arg1 (1:500; Cayman Chemical, Ann Arbor, MI, USA), anti-EGR2 (1:500; Cayman Chemical, Ann Arbor, MI, USA), anti-p85 (1:200; Abcam, Cambridge, MA, USA), anti-Akt and p-Akt (1:500; R\&D Systems, Minneapolis, MN, USA), anti-CD38 (1:100; Santa Cruz Biotechnology), anti-Fpr-2 (1:500; R\&D Systems, Minneapolis, MN, USA), anti-CD68 (1:100; Santa Cruz Biotechnology), and anti-active caspase-3 (1:500; Cayman Chemical), as described previously [12].

2.2.3. Bronchoalveolar Lavage Fluid (BALF) Preparation and Cell Counting. After 4, 12, 24, and $72 \mathrm{~h}$ of LPS stimulation ( $n=3$ per group at each time point), the lungs were gavaged three times with a total volume of $1.5 \mathrm{ml}$ cold PBS. Following centrifugation of the gavage fluid at $700 \mathrm{~g}$ for $10 \mathrm{~min}$ at $4^{\circ} \mathrm{C}$, the cell pellets were resuspended in $1 \mathrm{ml}$ of fresh PBS. Cell counting was performed using a hemocytometer in a doubleblind manner, while differential cell counting was done using Wright-Giemsa staining (KeyGen Biotech, Nanjing, China).

2.2.4. Assessment of Cytokines in Peripheral Blood. The concentrations of TNF- $\alpha$, IL- $1 \beta$, and IL-10 were measured in the peripheral blood collected from mice after 4, 12, 24, and $72 \mathrm{~h}$ ( $n=5$ per group at each time point) of LPS stimulation using ELISA kits (Abcam, Cambridge, UK). The optical density (OD) at $450 \mathrm{~nm}$ was used to assess the concentrations of each cytokine using GraphPad Prism 4.0 (GraphPad Software, Inc., San Diego, CA, USA).

2.2.5. Assessment of Wet-to-Dry Lung Weight Ratio (W/D Ratio). Lungs from each mouse were immediately dissected after sacrifice, and wet weight $(W)$ was recorded. Subsequently, the lung tissues were dried in an $80^{\circ} \mathrm{C}$ incubator for $48 \mathrm{~h}$, and later, dry weight $(D)$ was recorded. The $W / D$ ratio was calculated to assess lung edema [11].

2.2.6. Evans Blue Extravasation Method to Assess Pulmonary Vascular Permeability. Vascular permeability in each group was assessed using a modified Evans blue extravasation method. Briefly, mice ( $n=5$ from each group) were anesthetized with sodium pentobarbital $(60 \mathrm{mg} / \mathrm{kg}$, i.p.), and Evans blue dye ( $2 \%$ in $0.9 \%$ normal saline, $4 \mathrm{ml} / \mathrm{kg}$ ) was infused at $37^{\circ} \mathrm{C}$ via the right femoral vein within a 5 min time window. Two hours later, the mice were perfused with $300 \mathrm{ml}$ of normal saline to flush out any remaining dye in the blood vessels. Pulmonary vascular permeability was then evaluated in the lung tissues. To assess pulmonary vascular permeability, half of the lung tissue was removed and mechanically homogenized in $750 \mu \mathrm{l}$ of $N, N$-dimethylformamide (DMF; Sigma-Aldrich, St. Louis, MO). The suspended material was maintained at room temperature in the dark for $72 \mathrm{~h}$ and later centrifuged at $10,000 \times g$ for $25 \mathrm{~min}$. The supernatant was subsequently analyzed using a spectrophotometer (Molecular Devices OptiMax, USA) at $610 \mathrm{~nm}$ OD. The overall dye concentrations were expressed as $\mu \mathrm{g} / \mathrm{g}$ of tissue weight and calculated from a standard curve prepared using known concentrations of the dye [15]. The remaining left lung tissue was sectioned ( $20 \mu \mathrm{m}$ thick) for digital imaging.

2.2.7. Electron Microscopy (EM). Tissue sections were fixed in $10 \%$ glutaraldehyde and analyzed using a transmission electron microscope, as described previously [12].

2.2.8. Western Blot Analysis. The protein lysates from mouse lung tissue sections were prepared as described previously [15]. As a negative control, the membrane was probed in the absence of a primary antibody.

2.2.9. Statistical Analyses. All quantified results are presented as mean \pm standard deviation (SD). To compare groups, oneway analysis of variance (ANOVA) and the StudentNewman-Keuls (SNK) pairwise tests were used. A $P$ value of $<0.05$ represented statistical significance. All statistical analyses were conducted using SPSS 11.5 software package, and graphs were generated using GraphPad Prism Version 4.0 software (GraphPad Software, Inc. CA, USA).

\section{Results}

3.1. In Vitro Overexpression of TSP-2 Promotes M2 and TSP-1 Promotes M1 Macrophage Polarization. After LPS treatment (vehicle) of MH-S cells transfected with empty vector, the expression of M1 phenotype markers, CD38 and Fpr-2, increased compared to cells that were not treated (normal) (Figures 1(a), 1(b), 1(f), and 1(g)). In addition, LPS treatment of TSP-1-overexpressing MH-S cells did not alter CD38 and Fpr-2 expression (Figures 1(c) and 1(h)), but TSP-2 overexpression reduced CD38 and Fpr-2 expression compared to vehicle control (Figures 1(d) and 1(i)). The overall comparison of M1 markers, CD38 and Fpr-2, is shown in Figures 1(e) and $1(j)$.

Similarly, expression of M2 phenotype markers, Arg-1 and Egr-2, was reduced in LPS-treated MH-S cells compared to cells that were not treated (Figures 1(k), 1(l), 1(p), and $1(q))$. However, LPS treatment of TSP-1-overexpressing MH-S cells did not significantly change Arg-1 and Fpr-2 expression compared to vehicle-treated cells (Figures 1(m) and $1(\mathrm{r})$ ). Interestingly, LPS-treated TSP-2-overexpressing MH-S cells showed increased expression of Arg-1 and Egr2 (Figures $1(\mathrm{n})$ and $1(\mathrm{~s})$ ). The overall comparison of M2 phenotype markers, Arg-1 and Egr-2, is shown in Figures 1(o) and $1(\mathrm{t})$.

3.2. TSP-2 Overexpression Attenuates LPS-Induced Apoptosis and Promotes PI3K Signaling In Vitro. Next, we analyzed the 
CD38+Hoechst 33342 normal

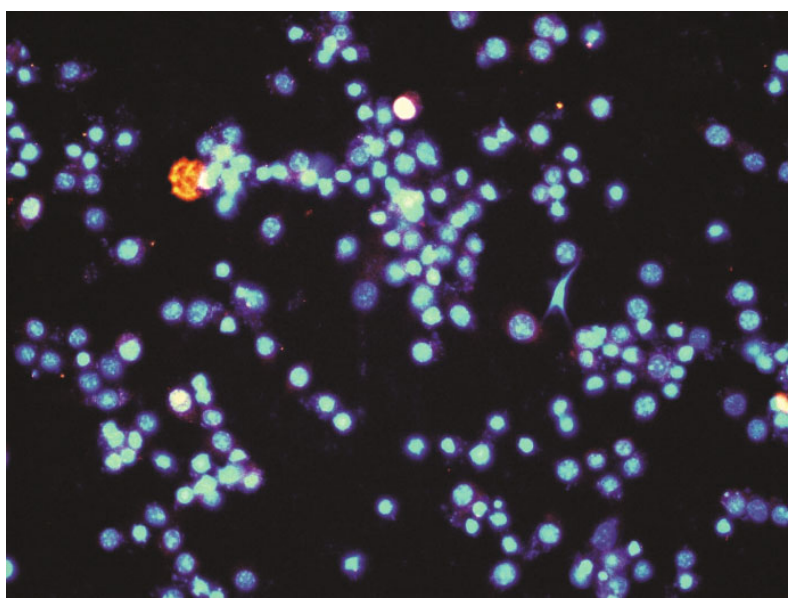

(a)

CD38 TSP-1

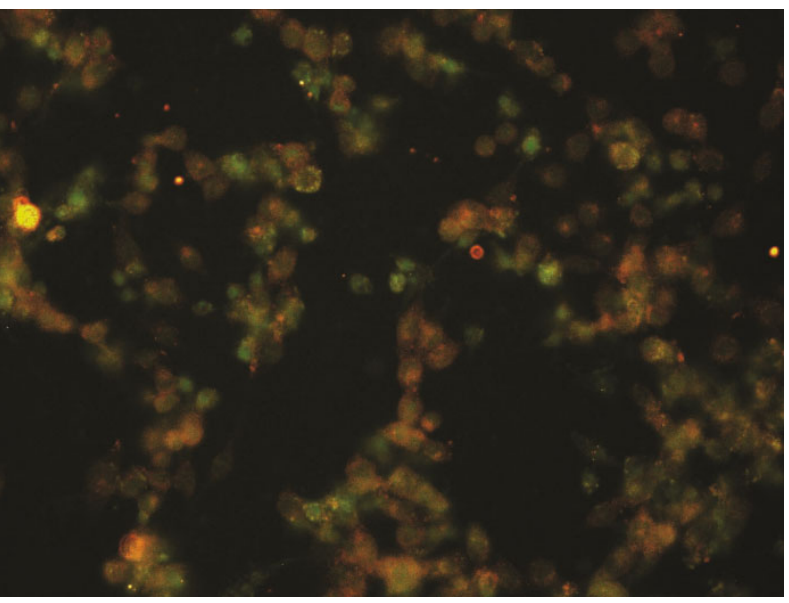

(c)

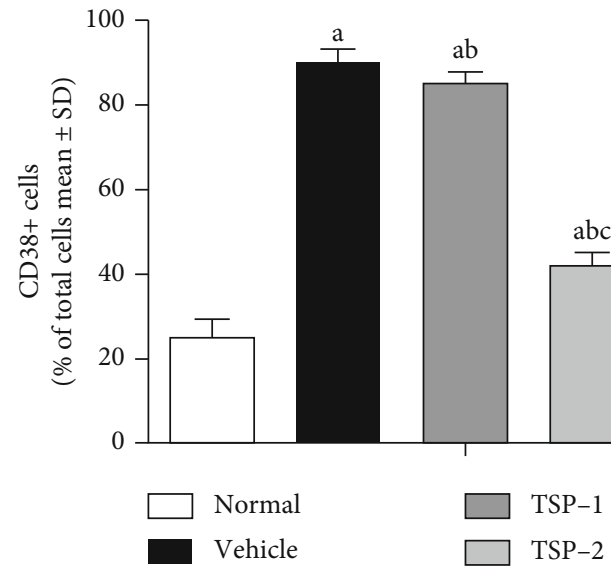

CD38+Hoechst 33342 vehicle

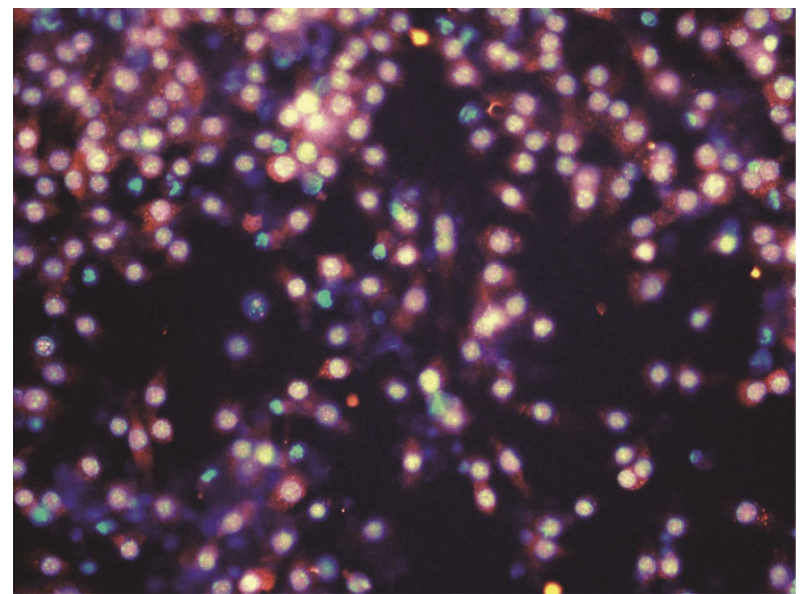

(b)

CD38 TSP-2

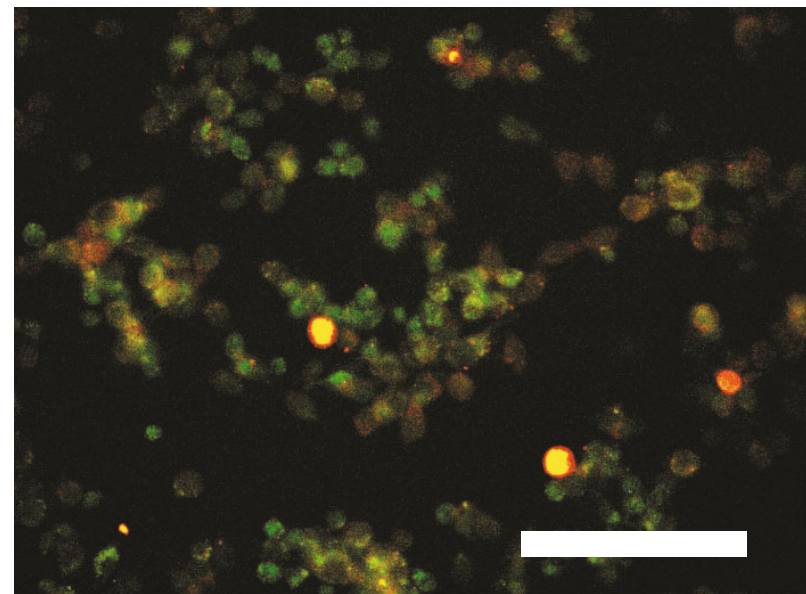

(d)

Fpr-2+Hoechst 33342 normal

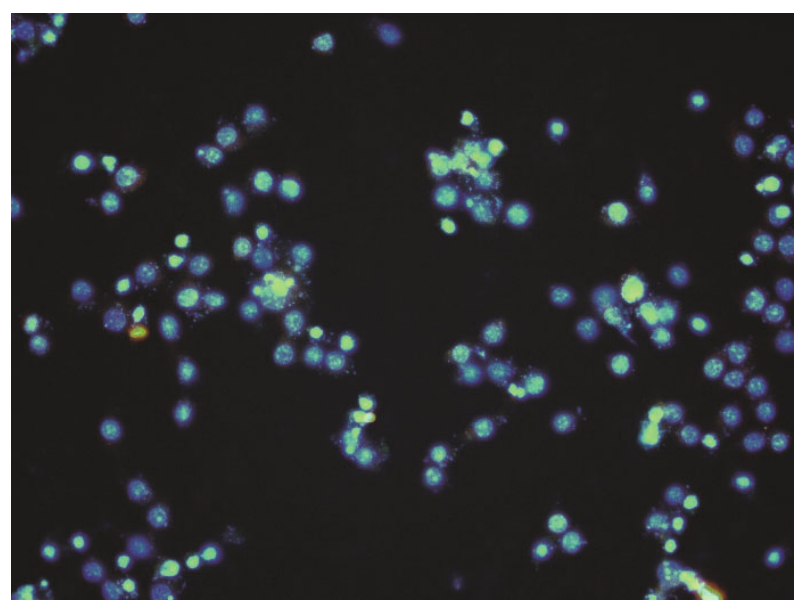

(f)

Figure 1: Continued. 
Fpr-2+Hoechst 33342 Vehicle

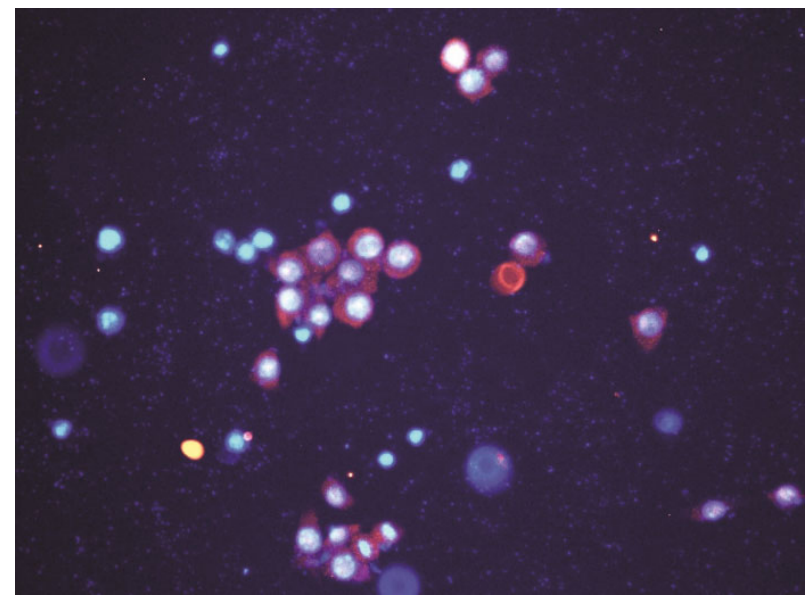

(g)

Fpr-2 TSP-2

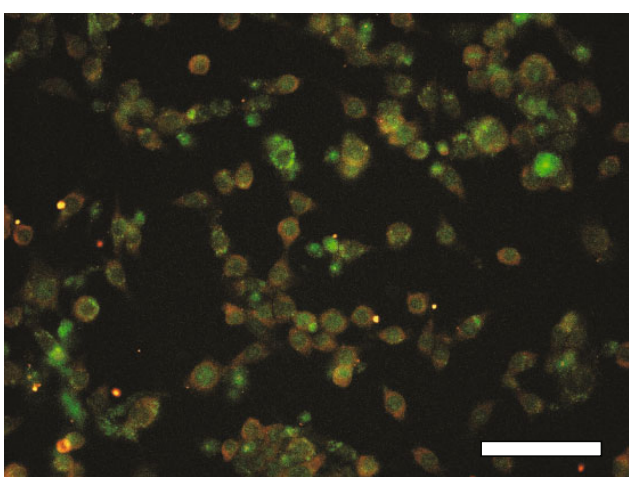

(i)

Arg-1+Hoechst 33342 normal

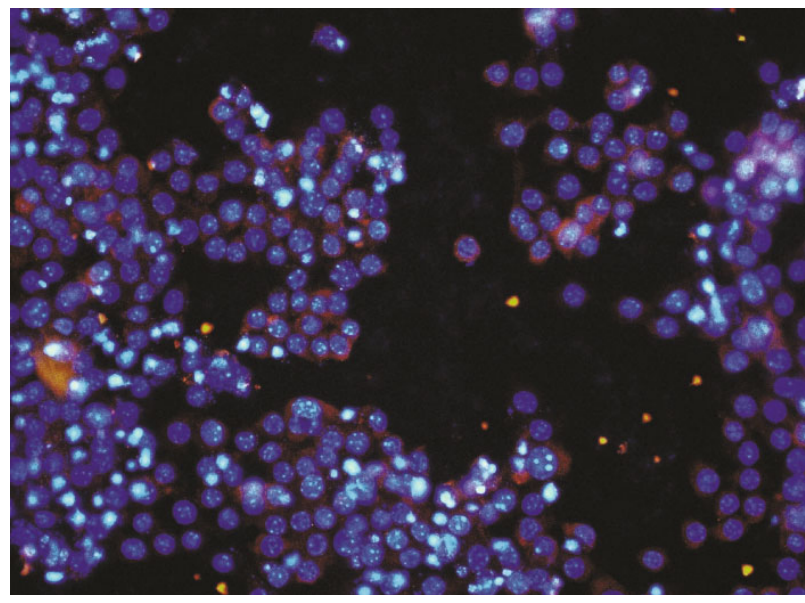

(k)
Fpr-2 TSP-1

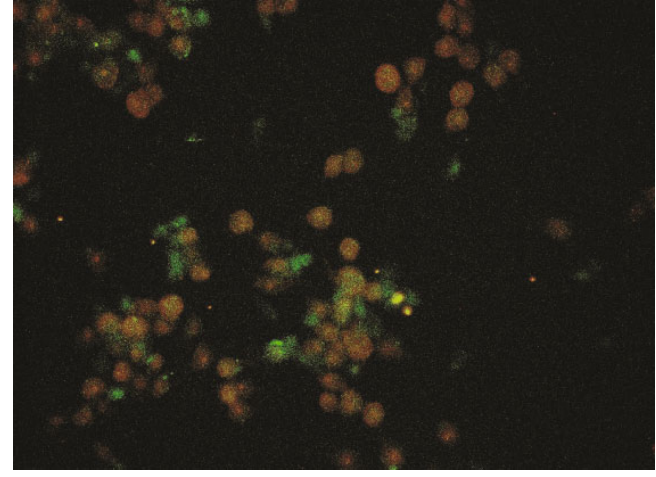

(h)

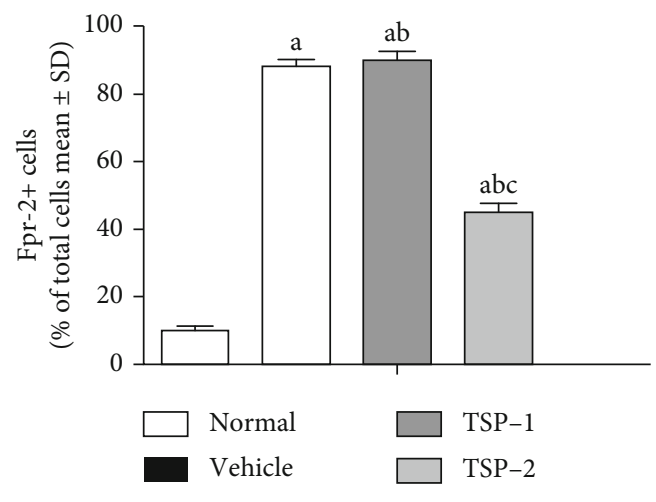

(j)

Arg-1+Hoechst 33342 vehicle

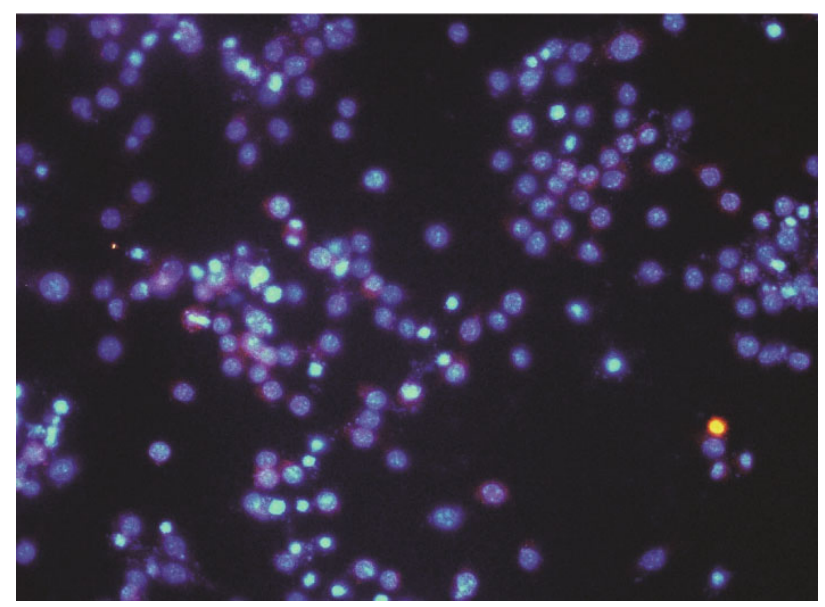

(1)

Figure 1: Continued. 
Arg-1 TSP-1

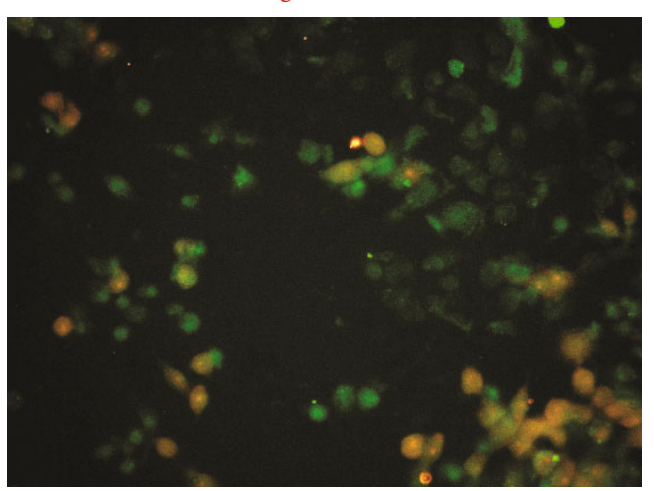

(m)

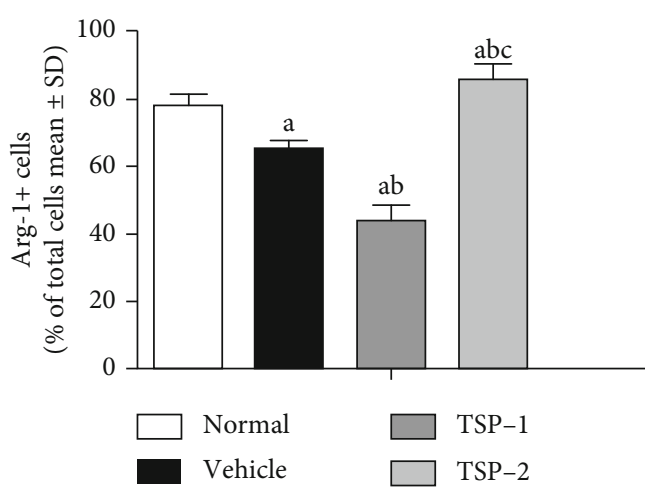

(o)

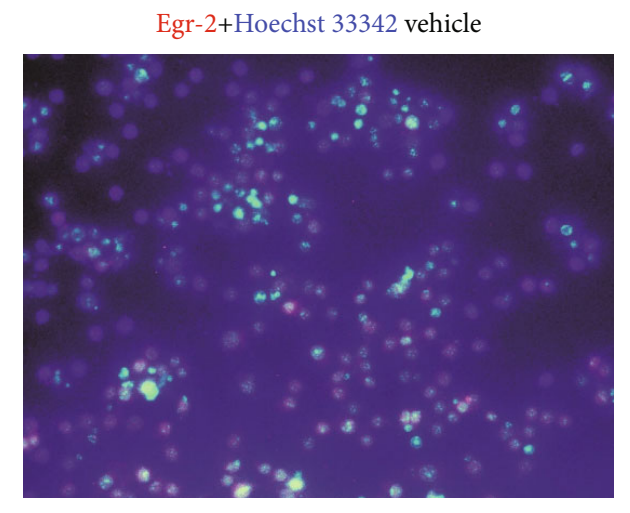

(q)
Arg-1 TSP-2

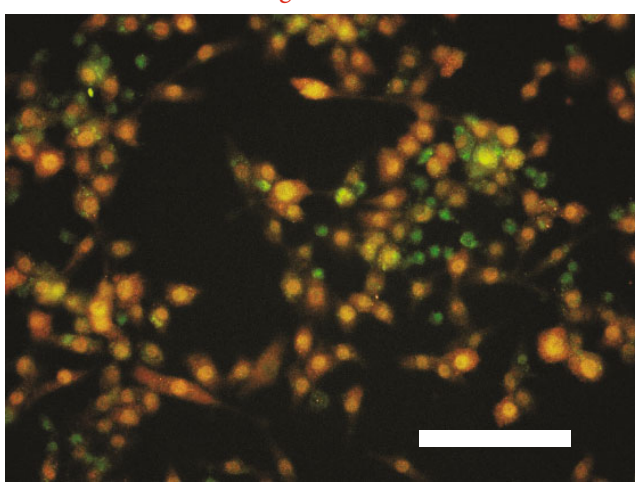

(n)

Egr-2+Hoechst 33342 normal

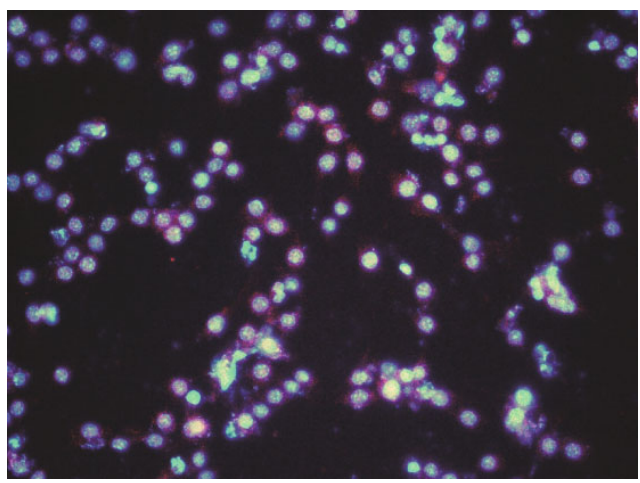

(p)

Egr-2 TSP-1

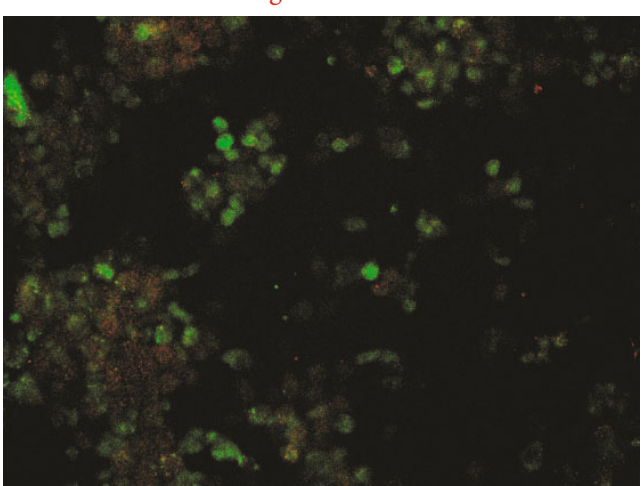

(r)

Figure 1: Continued. 


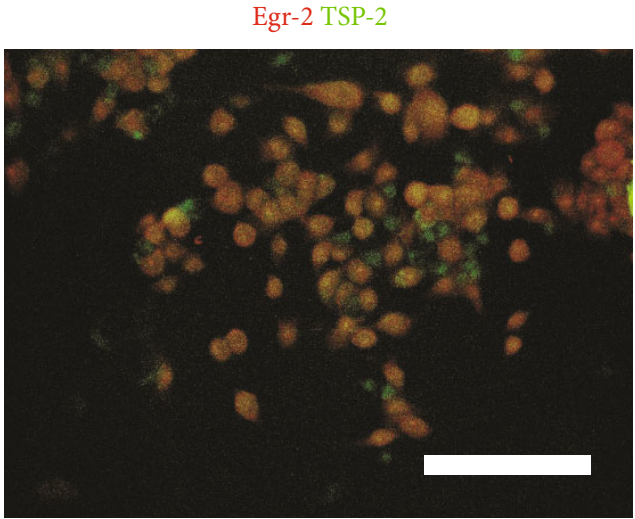

(s)

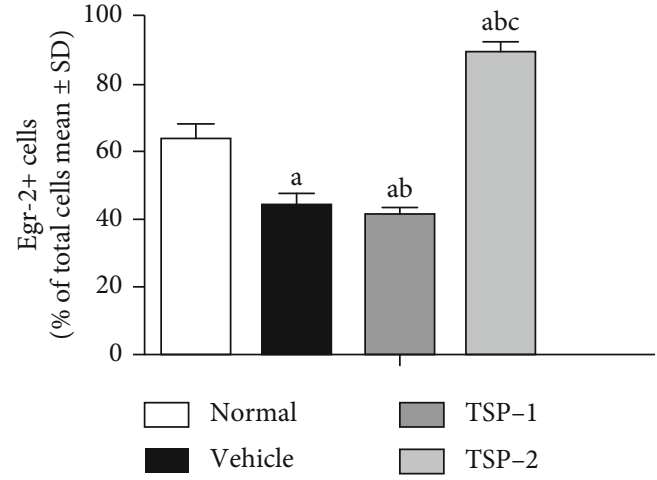

$(\mathrm{t})$

FIGURE 1: TSP-1 and TSP-2 overexpression affects macrophage polarization in vitro. MH-S murine alveolar macrophage cells were either untreated (normal and nontreatment) or treated with LPS $(1 \mu \mathrm{g} / \mathrm{ml})$ for $24 \mathrm{~h}$ following either empty vector (vehicle group), TSP-1 (TSP-1 group), or TSP-2 (TSP-2 group) overexpression. Cells were harvested and used for immunostaining of M1 (CD38 and Fpr-2) and M2 (Agr-1 and Egr-2) macrophage markers. (a-d) CD38 staining; (f-i) Fpr-2 staining; (k-n) Agr-1 staining; (p-s) Egr-2 staining in normal, vehicle, and TSP-1- and TSP-2-overexpressing cells, respectively. Red staining represents expression of specific markers, Hoechst 33342 blue staining represents the nuclei, and green staining represents GFP-labeled TSP-1 and TSP-2 overexpression. The yellow color depicts overlay image of specific markers and TSP expression in the same cell. Positively stained cells for specific markers in each group were counted and are shown in a bar graph in $(e, j, o$, and $t)$. The percentage of labeled cells/total cell numbers is shown as mean \pm SD. $P$ value of $<0.05$ represents significant difference. ${ }^{\text {a }} P<0.05$ compared with normal control; ${ }^{\mathrm{b}} P<0.05$ compared with vehicle group; ${ }^{\mathrm{c}} P<0.05$ compared with TSP-1 transfected group ( $n=5$ per group).

effects of TSP-1 and TSP-2 overexpression on apoptosis in MH-S cells after LPS induction by analyzing caspase-3 marker staining. Our data showed that LPS treatment itself induced the percentage of caspase-3-positive cells compared to no treatment (normal MH-S cells). However, TSP-1overexpressing MH-S cells after LPS treatment showed a similar percentage of caspase-3-positive staining, but TSP2-overexpressing MH-S cells showed a significantly reduced percentage of caspase-3-positive cells compared to LPStreated MH-S cells expressing empty vector (Figures 2(a)$2(d)$ ). Caspase- 3 staining in the four groups of cells is shown in Figure 2(e).

In addition, we analyzed the effects of TSP- 1 and TSP-2 overexpression on the PI3K signaling pathway in LPStreated MH-S cells. Specifically, we assessed expression of p85, p-Akt, and total Akt using immunostaining. As shown in Figure 3, LPS treatment alone reduced p85 signal intensity (Figure 3(b)), while TSP-1 and TSP-2 overexpression increased the p85 signal (Figures 3(c) and 3(d)). Quantification of p85 staining in the different conditions is shown in Figure 3(e). Similarly, LPS treatment reduced the levels of total Akt, but TSP-1 and TSP-2 overexpression restored Akt levels (Figures 3(f)-3(j)). Moreover, p-Akt expression decreased after LPS treatment (Figure 3(l)), but only TSP-2 overexpression restored p-Akt expression (Figure 3(n)), while TSP-1 overexpression had no effect (Figure 3(m)). The bar graph in Figure 3(o) shows the overall signal intensity of p-Akt among the four groups.

3.3. TSP-1 and TSP-2 Overexpression Differentially Affects Cytokine Secretion in the ARDS Mouse Model. Macrophages from mice injected with TSP-1-overexpressing cells showed significant upregulation of TSP-1, as expected. The bar graphs in Supplementary Figure 1B present the parallel comparison of TSP-1 expression among mice in the different groups. Supplementary Figures 1C and 1D show the upregulation of TSP-2 only in mice injected with TSP-2-overexpressing cells. We next analyzed the serum cytokine levels in the four groups (Figures 4(a)-4(c)). IL-6, TNF- $\alpha$, and IL-10 were induced in mice that were injected with TSP-1 similar to LPS treatment alone. However, TSP-2 overexpression significantly suppressed LPS-induced secretion of inflammatory cytokines IL- 6 and TNF- $\alpha$, but upregulated secretion of antiinflammatory factor IL-10.

3.4. TSP-2 Overexpression Inhibits Pulmonary Vascular Permeability and Edema in ARDS Mice. We next assessed the effects of TSP proteins on pulmonary vascular permeability using Evan's blue extravasation assay in the ARDS mouse model. We observed that TSP-2 overexpression significantly reduced pulmonary vascular permeability, as evident from reduced staining (red color) due to poor leakage (Figures 5(c), 5(f), 5(i), and 5(l)) compared to LPS-treated mice transfected with empty vector, which had higher staining (red color) due to increased pulmonary vessel permeability at all time points (Figures 5(a), 5(d), 5(g), and 5(j)). However, TSP-1 overexpression resulted in higher pulmonary vascular permeability compared to the TSP-2 overexpression group. Figure 5(m) shows the basal level of staining in normal mice without any LPS treatment. The bar graphs in Figure 5(n) represent the normalized dye leakage in the different experimental conditions. Similarly, we assessed the effects of TSP overexpression on pulmonary edema by measuring the $W / D$ ratio of lungs in the ARDS mice. As shown in Figure 4(d), TSP-2 overexpression reduced the $W / D$ ratio, while TSP-1 overexpression had similar effects compared to LPS treatment alone. 
Caspase-3+Hoechst 3342 normal

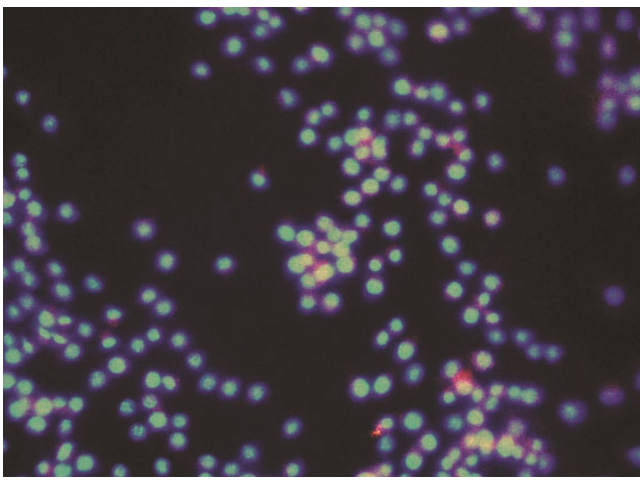

(a)

Caspase-3 TSP-1

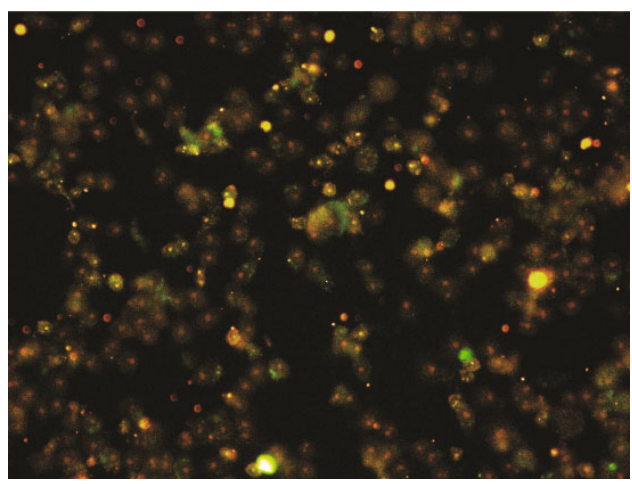

(c)
Caspase-3+Hoechst 33342 vehicle

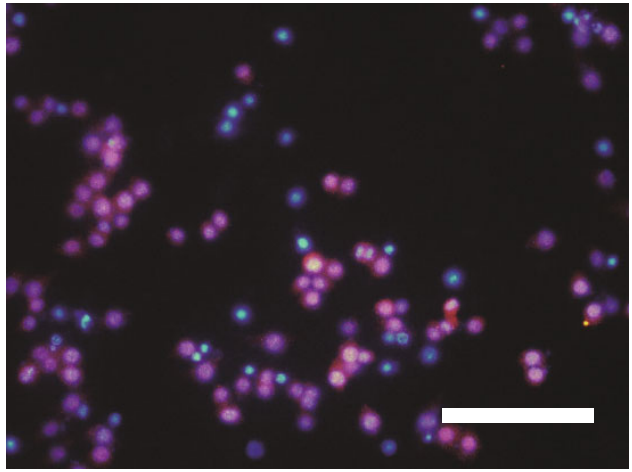

(b)

Caspase-3 TSP-2

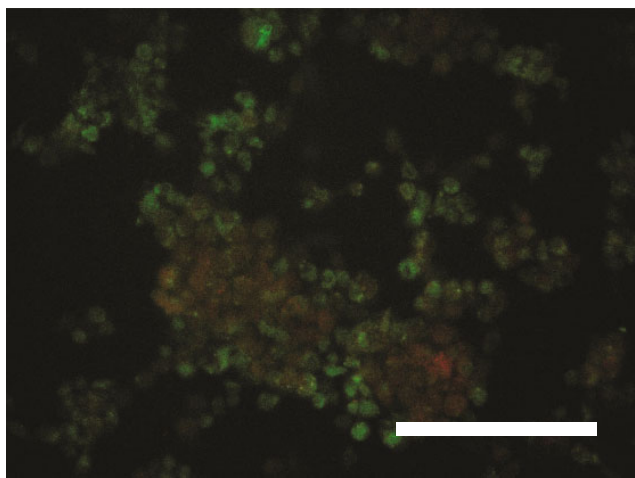

(d)

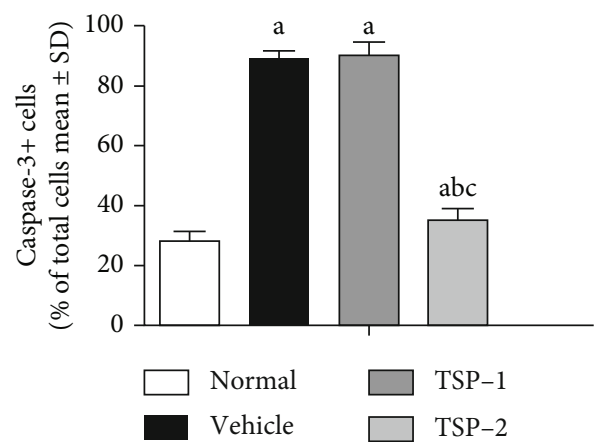

(e)

FIGURE 2: Effect of TSP-2 overexpression on LPS-induced apoptosis. MH-S murine alveolar macrophage cells were either untreated (normal and nontreatment) or treated with LPS $(1 \mu \mathrm{g} / \mathrm{ml})$ for $24 \mathrm{~h}$ following either empty vector (vehicle group), TSP-1 (TSP-1 group), or TSP-2 (TSP2 group) overexpression. Cells were stained with caspase-3 (red) antibody to assess apoptosis (a-d), respectively. Hoechst 33342 dye (blue) was used to stain the nucleus. Green stain represents TSP-1 and TSP-2 overexpression. The yellow color depicts overlay image of caspase3 and TSP expression in the same cell. (e) Bar graph representing overall quantitation and parallel comparison of caspase- 3 staining among the four groups. The percentage of labeled cells/total cell numbers are shown as mean $\pm \mathrm{SD}$. $P$ value of $<0.05$ represents significant difference. ${ }^{\mathrm{a}} P<0.05$ compared with normal control; ${ }^{\mathrm{b}} \mathrm{P}<0.05$ compared with the vehicle group; ${ }^{\mathrm{c}} \mathrm{P}<0.05$ compared with the TSP-1 transfected group ( $n=5$ per group).

3.5. TSP-2 Overexpression Inhibits Pulmonary Inflammation and Lung Injury in ARDS Mice. To understand the role of TSP proteins in pulmonary inflammation, we analyzed the expression of CD45, a specific marker of leukocytes in lung tissue, using Western blot analysis. CD45 expression increased in lung tissue sections after LPS stimulation in the vector and TSP-1-overexpressing mice, but significantly decreased in the TSP-2-overexpressing mice, as shown in Figures 6(a) and 6(b).

We also analyzed the accumulation of inflammatory cells in the BALF of mice from the four different groups using Wright-Giemsa staining. We observed neutrophil accumulation after $4,12,24$, and $72 \mathrm{~h}$ of LPS treatment in mice expressing empty vector. A similar neutrophil 
P85+Hoechst 33342 normal

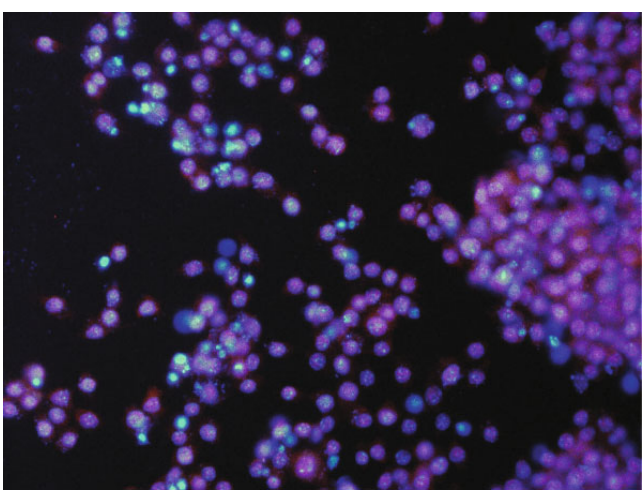

(a)

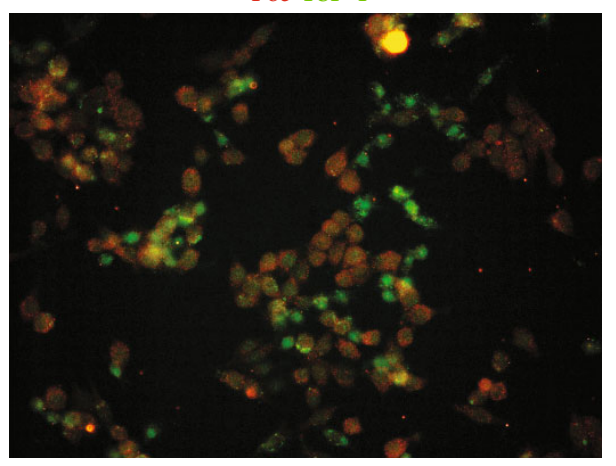

(c)

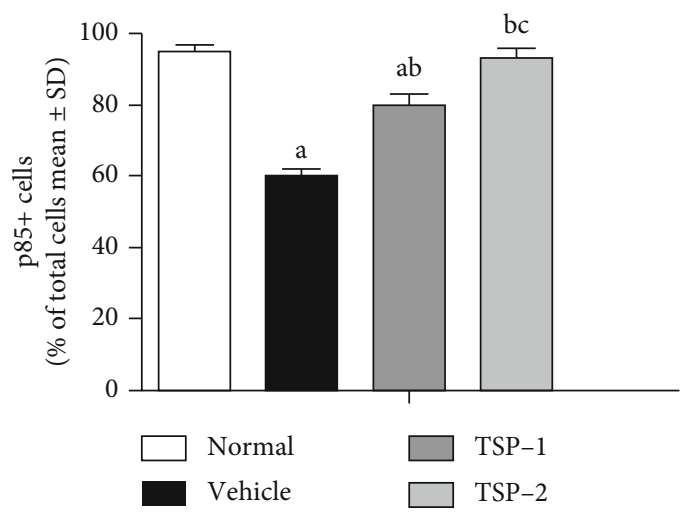

(e)

Akt+Hoechst 33342 vehicle

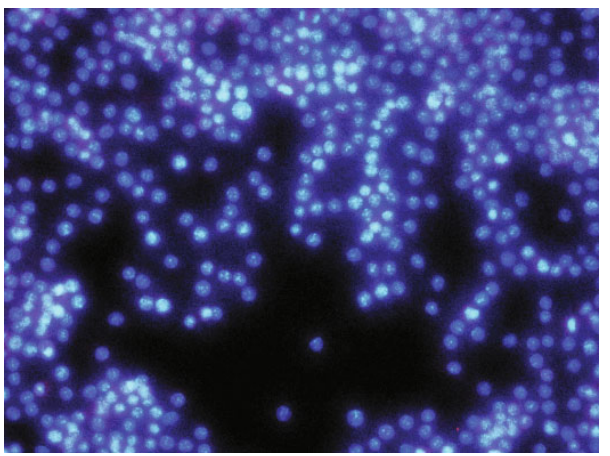

(g)
P85+Hoechst 33342 vehicle

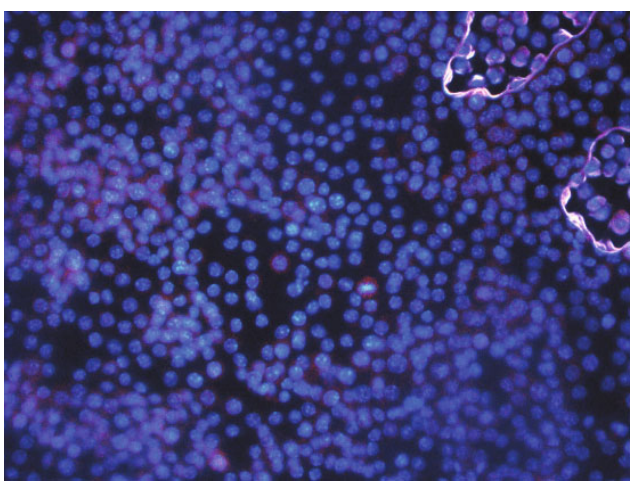

(b)

p85 TSP-2

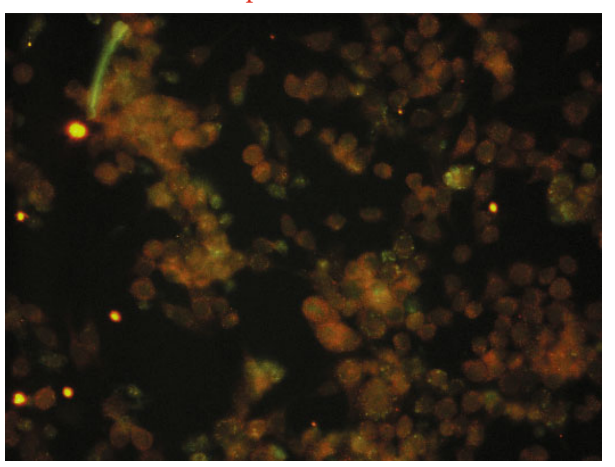

(d)

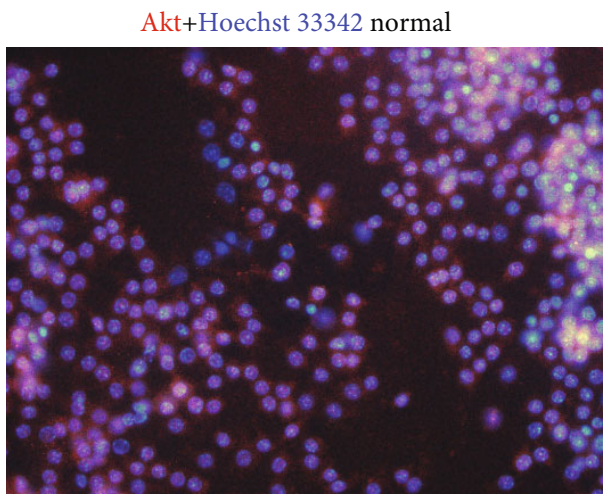

(f)

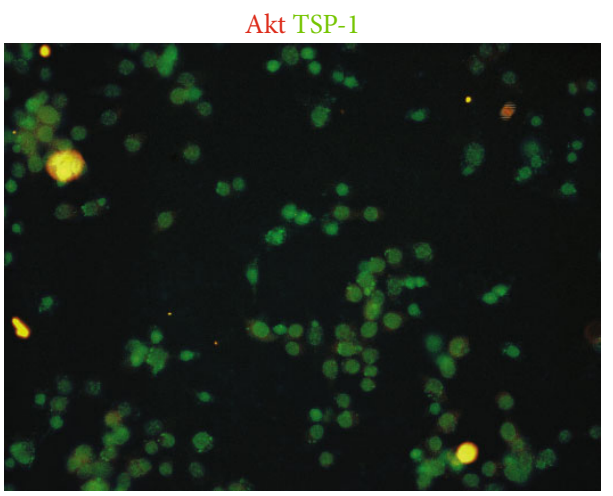

(h)

Figure 3: Continued. 


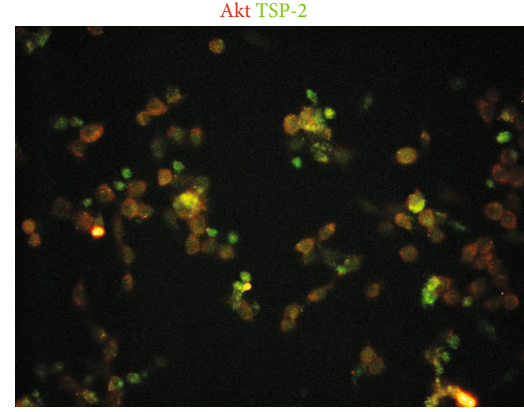

(i)

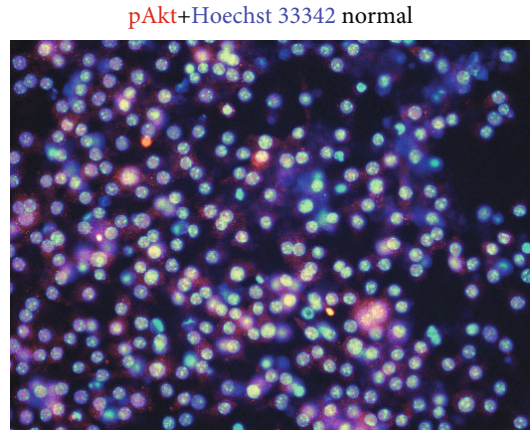

$(\mathrm{k})$

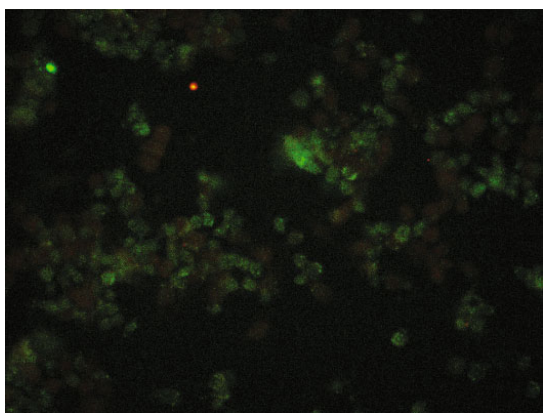

$(\mathrm{m})$

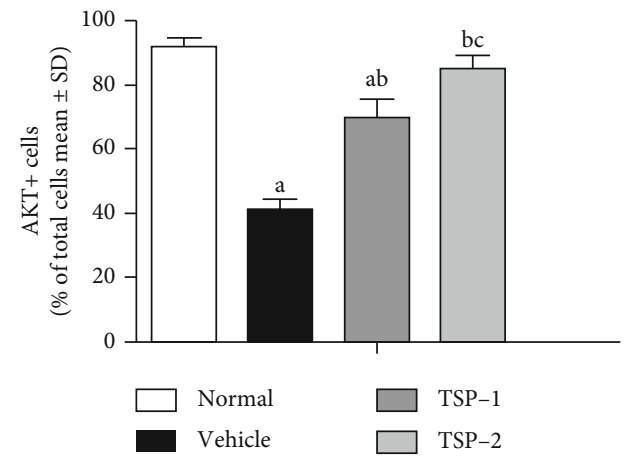

(j)

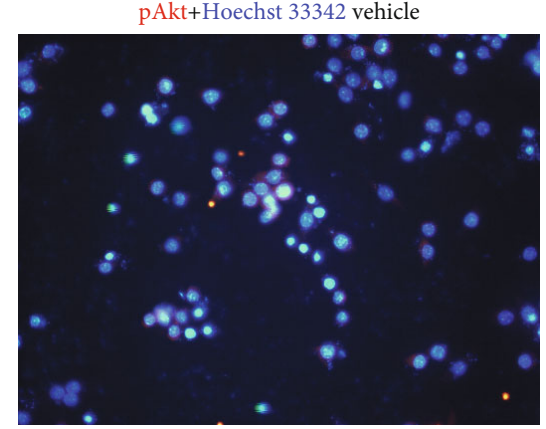

(l)

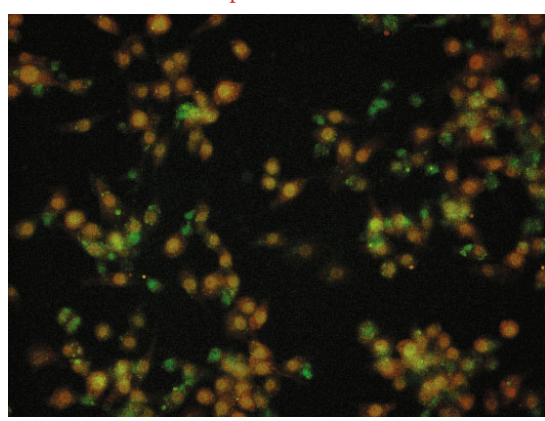

(n)

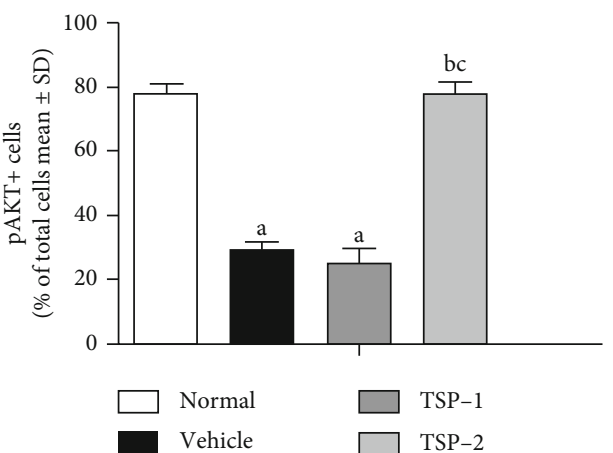

(o)

FIGURE 3: Impact of TSP-2 overexpression on PI3K signaling in vitro. MH-S murine alveolar macrophage cells were either untreated (normal and nontreatment) or treated with LPS $(1 \mu \mathrm{g} / \mathrm{ml})$ for $24 \mathrm{~h}$ following either empty vector (vehicle group), TSP-1 (TSP-1 group), or TSP-2 (TSP-2 group) overexpression. MH-S cells were stained with p85 (a-d), total Akt ( $\mathrm{f}-\mathrm{i})$, and $\mathrm{p}-\mathrm{Akt}(\mathrm{k}-\mathrm{n})$ antibodies to assess the impact on PI3K signaling. Red color is antibody-specific staining, blue color is Hoechst 33342 nuclei staining, and green color is GFP-labeled TSP-1and TSP-2-overexpressing cells. Yellow color shows colocalization of TSP-1 or TSP-2 with specific proteins. (e, j, and o) Bar graphs showing parallel comparisons of antibody staining quantitation among the four groups. The percentage of labeled cells/total cell numbers is shown as mean \pm SD. $P$ value of $<0.05$ represents significant difference. ${ }^{a} P<0.05$ compared with normal control; ${ }^{b} P<0.05$ compared with the vehicle group; ${ }^{\mathrm{c}} P<0.05$ compared with the TSP-1 transfected group ( $n=5$ per group).

accumulation profile was observed in the BALF collected from TSP-1-overexpressing mice, but the BALF from TSP-2overexpressing mice displayed a significantly reduced number of neutrophils (Supplementary Fig 2A-N).

Next, we investigated pulmonary morphology in ARDS mice from four different groups using H\&E staining of lung tissues. We observed alveolar septa thickening and inflammatory cell infiltration after $4 \mathrm{~h}$ of LPS treatment in the empty vector and TSP-1-expressing mice (Figures 7(a) and $7(\mathrm{~b})$ ), and more severe alveolar hemorrhage and additional thickening were evident at later time points $(12,24$, and $72 \mathrm{~h}$ ) (Figures $7(\mathrm{~d}), 7(\mathrm{e}), 7(\mathrm{~g}), 7(\mathrm{~h}), 7(\mathrm{j})$, and $7(\mathrm{l})$ ). In contrast, 


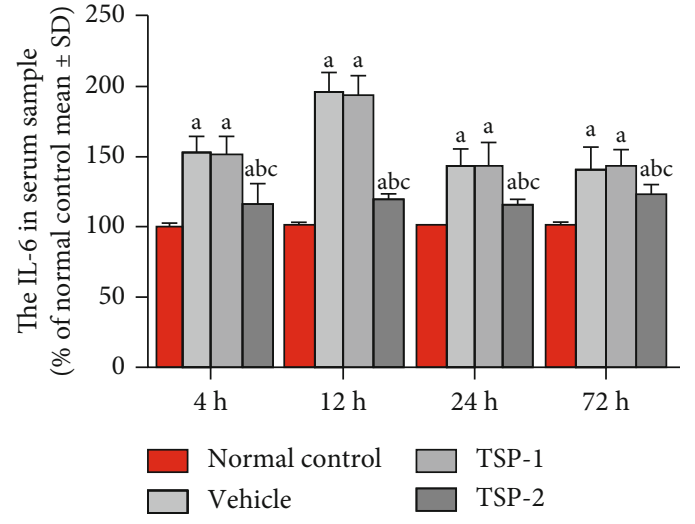

(a)

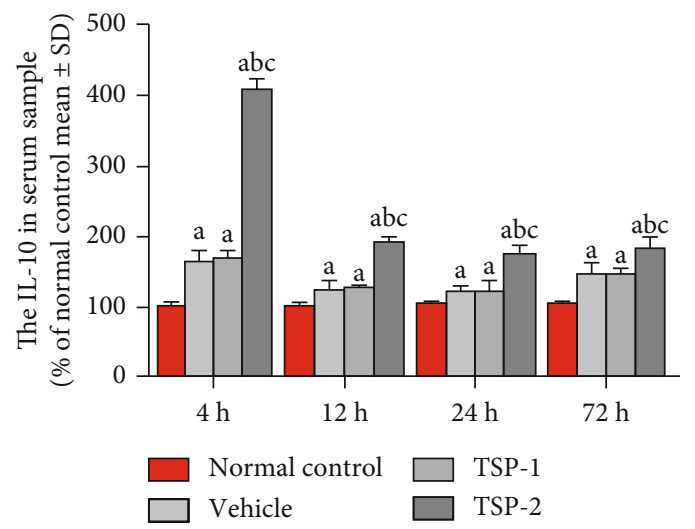

(c)

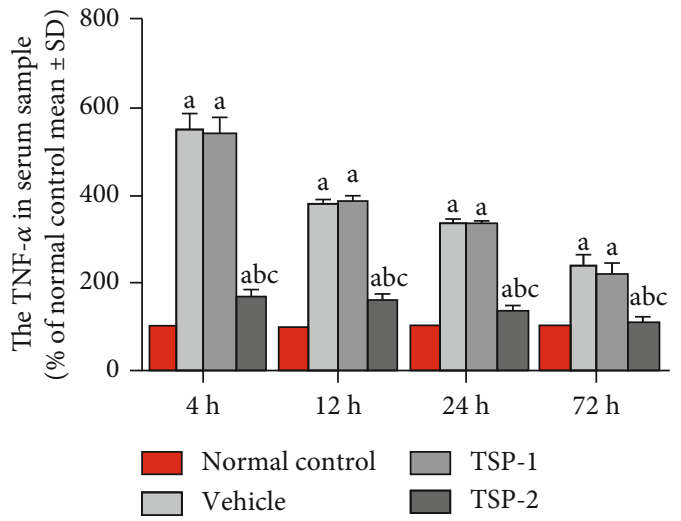

(b)

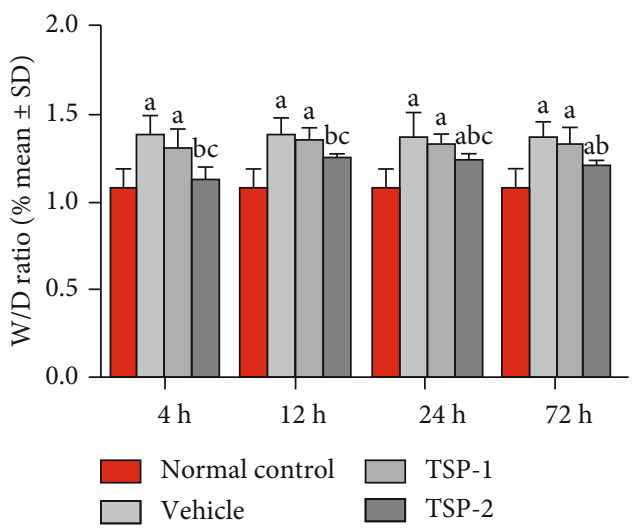

(d)

FIGURE 4: ELISA analyses of inflammatory cytokines in mouse serum and assessment of lung W/D ratios. (a-c) Bar graphs representing inflammatory cytokines IL-6 (a), TNF- $\alpha$ (b), and IL-10 (c) in serum from the normal, vehicle, TSP-1, and TSP-2 groups, using ELISA. (d) Comparison of lung $W / D$ ratio among the four groups. (a-c) The percentage of expression level of each group/normal control is shown as mean \pm SD. (d) The lung $W / D$ ratio of each group is shown as mean \pm SD. $P$ value of $<0.05$ represents significant difference. ${ }^{\text {a }} P<0.05$ compared with normal control; ${ }^{\mathrm{b}} P<0.05$ compared with the vehicle group; ${ }^{\mathrm{c}} P<0.05$ compared with the TSP-1 transfected group $(n=5$ per group).

TSP-2-overexpressing mice displayed reduced alveolar hemorrhage and septa thickening, as well as less inflammatory cell infiltration at all time points after LPS treatment (Figures 7(c), 7(f), 7(i), and 7(l)). Lung tissue injury scores from mice are shown in Figure $7(n)$. The effects of TSP-2 on pulmonary histology were consistent with the observed decrease in lung inflammation.

Additional ultrastructural morphology analysis of the pulmonary tissue sections from normal mice (no treatment) using EM revealed normal blood vessel epithelial cells (Figure 8(a)), air-blood barrier with a thin basement membrane (Figure 8(b)), macrophages in the blood vessels (Figure 8(c)), and alveolar epithelial cells (AECs) with clear lamellar bodies (Figure 8(d)). However, lung sections from LPS-treated mice showed disruption of the basement membrane in the capillaries (Figure 8(e)); thickened air-blood barrier (Figure $8(\mathrm{f})$ ); perivascular edema and inflammatory cell infiltration (Figure 8(g)); and apoptotic AECs with visible empty lamellar bodies, swollen cell organs, and contracted nuclei with condensed and fragmented nuclear chromatin (Figure $8(\mathrm{~h})$ ). A similar trend was observed in lung sections from TSP-1-overexpressing mice (Figures 8(i)-8(1)). However, lung sections from TSP-2-overexpressing mice after LPS treatment displayed relatively normal ultrastructural morphology of blood vessel epithelial cells (Figure $8(\mathrm{~m})$ ), air-blood barrier (Figure 8(n)), and alveolar epithelial cells (Figure $8(\mathrm{p})$ ). In addition, we observed attenuated perivascular edema in these sections (Figure 8(o)).

3.6. TSP-2 Overexpression Diminishes Pulmonary Apoptosis in ARDS Mice. Since TSP-2 suppressed LPS-induced apoptosis in vitro, we next analyzed its effects in vivo by measuring caspase-3 expression using Western blot analysis and immunofluorescence staining of lung tissue sections from the four groups. Our results revealed that TSP-2 overexpression suppressed expression of active caspase- 3 in pulmonary tissue compared to LPS-treated mice expressing empty vector or TSP-1 overexpression (Figures 6(c) and 6(d)). Immunofluorescence staining analysis further illustrated that lung tissues of LPS-treated mice with empty vector or TSP-1 overexpression had more caspase-3-positive cells (Supplementary Fig $3 \mathrm{~A}, \mathrm{~B}, \mathrm{D}, \mathrm{E}, \mathrm{G}, \mathrm{H}, \mathrm{J}, \mathrm{K}$, and $\mathrm{N}$ ), while lung sections from 

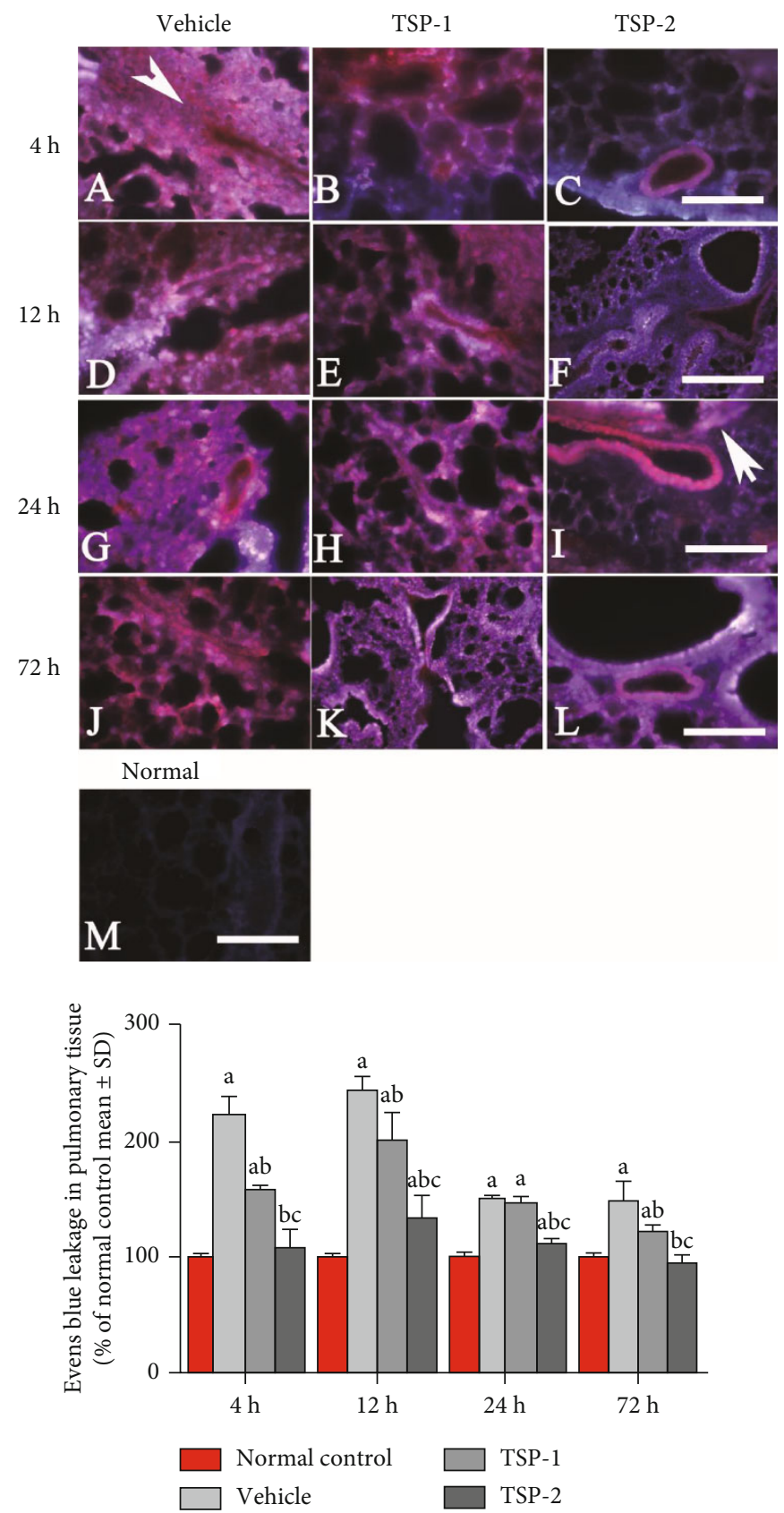

$(\mathrm{N})$

FIGURE 5: The impact of TSP-2 on pulmonary vascular permeability in the ARDS mouse model. (a-l) Evans blue (EB) staining is shown as an index of pulmonary vascular permeability in lung sections collected from vehicle, TSP-1, and TSP-2-overexpressing mice. (m) Normal mice. (n) Percentage of EB leakage in pulmonary tissue compared to normal control. EB staining was visualized using red laser excitation at $405 \mathrm{~nm}$ OD. Blue color represents no EB leakage into the pulmonary tissue, red color depicts high EB leakage due to enhanced vascular permeability, and pink color shows medium vascular permeability. The arrows point to the site of EB leakage. Scale bar $=100 \mu \mathrm{m}$ and $n=5$. (n) Evans blue leakage in pulmonary tissue $(\mu \mathrm{g} / \mathrm{g})$ is shown as mean \pm SD. $P$ value of $<0.05$ represents significant difference. ${ }^{\mathrm{a}} P<0.05$ compared with normal control; ${ }^{b} P<0.05$ compared with the vehicle group; ${ }^{c} P<0.05$ compared with the TSP- 1 transfected group ( $n=4$ per group).

TSP-2-overexpressing mice and normal control mice exhibited diffuse caspase-3 staining (Supplementary Fig 3C, F, I, $\mathrm{L}, \mathrm{M}$, and $\mathrm{O}$ ).

3.7. TSP-2 Overexpression Induces M2 Macrophage Phenotype through Activation of PI3K Signaling In Vivo. We next analyzed the effect of TSP proteins on macrophage polarization and PI3K signaling in ARDS mice. Protein analysis of lung tissue lysates from mice in the four groups showed that expression of M2 phenotype markers, Egr-2 (Figures 9(a) and 9(b)) and Arg-1 (Figures 9(c) and 9(d)), increased significantly in the TSP-2-overexpressing mice at all time points after LPS treatment, while expression of M1 phenotypespecific markers, CD38 (Figures 9(e) and 9(f)) and Fpr-2 


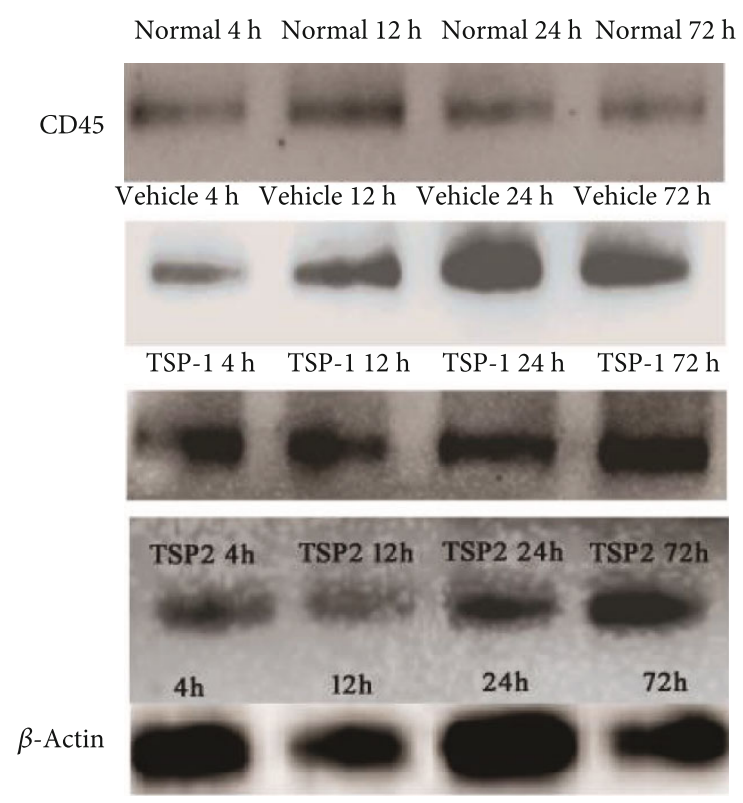

(a)

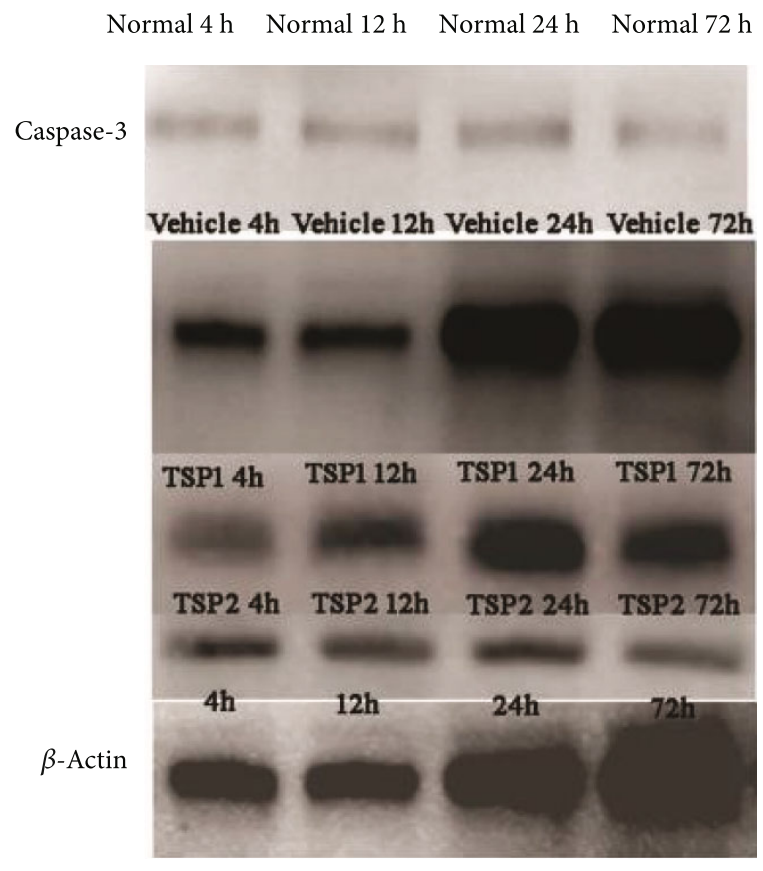

(c)

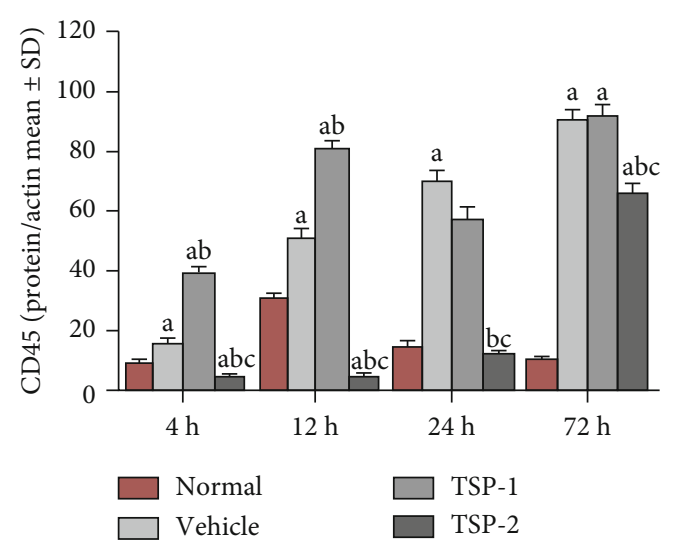

(b)

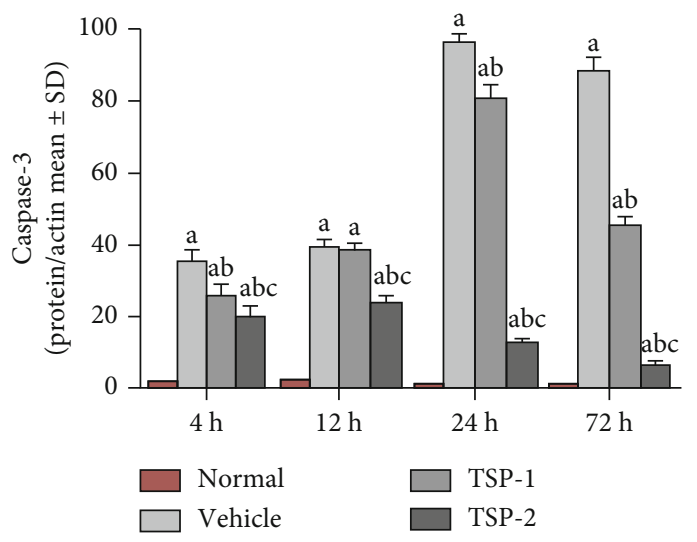

(d)

FIGURE 6: CD45 and caspase-3 protein expression in lung tissue lysates. Mouse protein lysates from normal mice or mice treated with LPS for $4,12,24$, and $72 \mathrm{~h}$ and overexpressing either empty vector (vehicle group), TSP-1 (TSP-1 group), or TSP-2 (TSP-2 group) were analyzed for CD45 (a) and caspase-3 (c) protein expression using Western blot analysis. $\beta$-Actin expression served as the loading control. (b, d) Bar graphs representing CD45 and caspase- 3 expression after normalizing to $\beta$-actin expression among four different groups. The percentage of detected protein/actin is shown as mean \pm SD. $P$ value of $<0.05$ depicts significant difference. ${ }^{a} P<0.05$ compared with normal control; ${ }^{b} P<0.05$ compared with vehicle group; ${ }^{\mathrm{c}} P<0.05$ compared with TSP-1 transfected group ( $n=4$ per group).

(Figures $9(\mathrm{~g})$ and $9(\mathrm{~h})$ ), increased in TSP-1-overexpressing mice. In vivo analysis of the effect of TSP proteins on PI3K signaling showed a similar pattern to the in vitro analysis. TSP-1-overexpressing and vehicle lung tissue lysates showed reduced p85, p-Akt, and total Akt protein levels compared to normal control (Figures 10(a)-10(f)). However, TSP-2overexpressing lung tissue lysates showed no significant change, and their protein levels were similar to control lysates, which suggested that TSP-2 overexpression reversed the negative effects of LPS on this signaling pathway. In vivo inhibition of the PI3K/Akt pathway using LY294002 resulted in a significant increase in CD68 expression in the TSP-2-transfected mice compared to the control mice. Additionally, these mice showed no remarkable differences in CD68 expression 

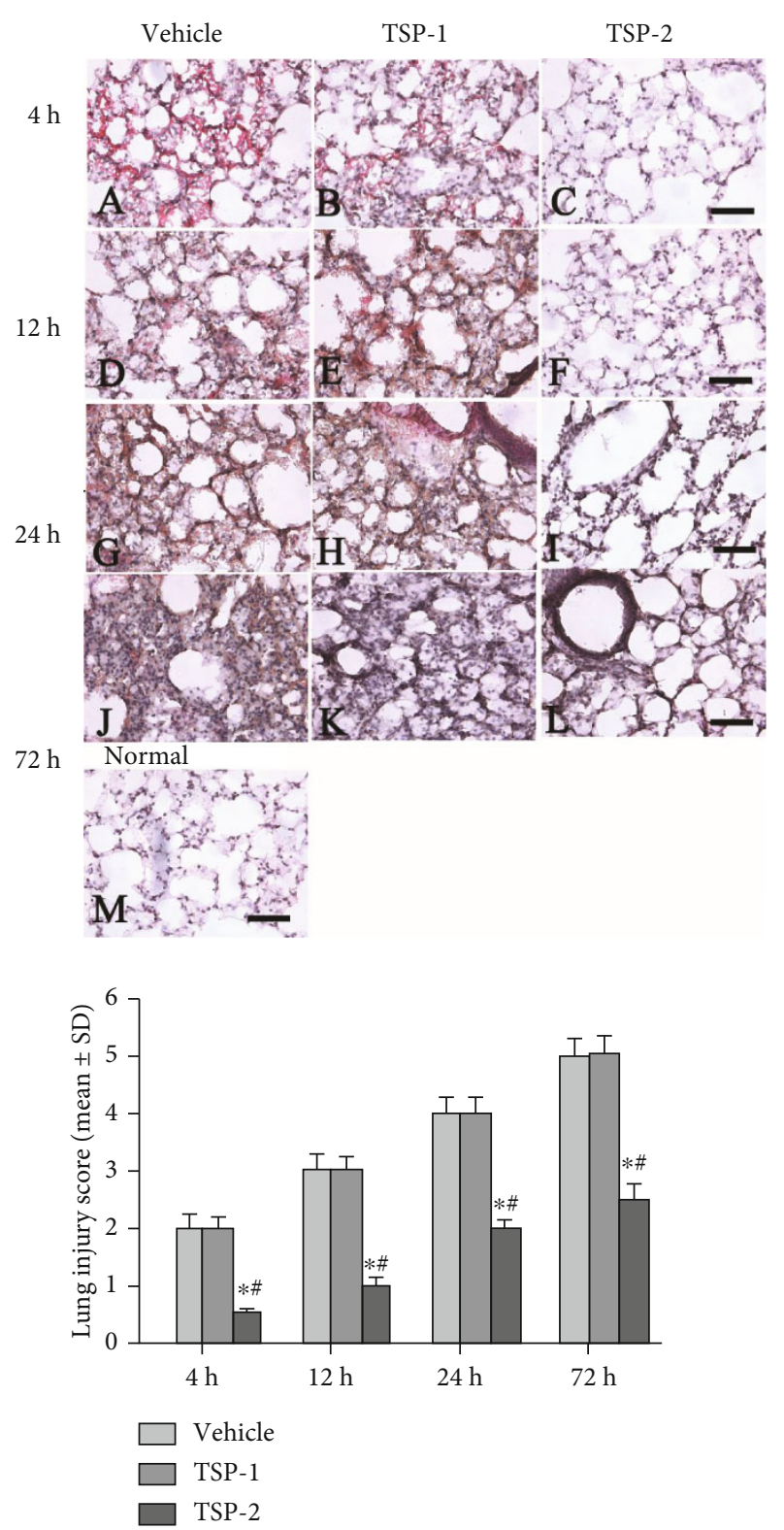

$(\mathrm{N})$

FIgURE 7: Effect of TSP-2 overexpression on pulmonary morphology in the ARDS mouse model. Lung tissue sections from mice treated with LPS for $4,12,24$, and $72 \mathrm{~h}$ and overexpressing either empty vector (vehicle group), TSP-1 (TSP-1 group), or TSP-2 (TSP-2 group) were analyzed for pulmonary morphology using H\&E staining (a-l). (m) Normal control: the normal morphology of blood vessels, bronchi, and interstitium. Scale bar $=100 \mu \mathrm{m}$. (n) Bar graph of lung injury scores from three different groups, assessed by two independent pathologists blinded to the experimental conditions. Scoring was based on the following: $0=$ no injury; 1 = edema/fibrin, hemorrhage (subpleural); $2=$ edema/fibrin, hemorrhage (interlobular); $3=$ edema/fibrin, hemorrhage (alveolar); $4=$ congestion of alveolar septa; $5=$ hyaline membrane changes of alveolar septa. The lung injury score of each group is shown as mean \pm SD. $P$ value of $<0.05$ represents significant difference. ${ }^{\mathrm{a}} P<0.05$ compared with normal control; ${ }^{\mathrm{b}} P<0.05$ compared with the vehicle group; ${ }^{\mathrm{c}} P<0.05$ compared with the TSP- 1 transfected group ( $n=5$ per group). compared to the mice in the vehicle and TSP-1-transfected groups. CD68 expression was exacerbated in the TSP-1transfected mice treated with LY294002 (Figure 11).

\section{Discussion}

In this study, we sought to delineate the role of TSP proteins in LPS-induced ARDS. Among the two TSP proteins that were analyzed, TSP-2 overexpression specifically and effectively attenuated LPS-induced ARDS by inhibiting apoptosis, pulmonary vascular permeability, edema, inflammation, and lung injury through inducing the PI3K signaling pathway. Furthermore, we observed that TSP-2 overexpression favored M2 macrophage polarization both in vitro and in vivo. In contrast, TSP-1 overexpression was not able to reverse the LPSmediated effects in either the in vitro or in vivo model, and TSP-1 favored M1 macrophage polarization.

4.1. TSP-1. TSP-1 is a matricellular protein that has been described as an important factor in maintaining vascular structure and homeostasis through regulating various biological functions, such as cell proliferation, apoptosis, and adhesion [16]. Some studies have indicated that TSP-1 can promote monocyte migration to the injury site [17], where it typically promotes monocytic cell adhesion to extracellular matrix (ECM). Thus, TSP-1 expression plays a significant role in mononuclear cell migration and adhesion, and subsequently contributes to vascular inflammation. It has also been reported that macrophages derived from TSP-1-deficient mice had a reduced inflammatory phenotype, thereby indicating the role of TSP-1 in macrophage activation [18]. In the same study, it was demonstrated that human TSP-1 treatment stimulated TNF- $\alpha$ expression in bone marrow-derived macrophages in a time- and dose-dependent manner, and TSP-1stimulated macrophage activation was TLR4 dependent [18]. Another study demonstrated that TSP-1 can act as a positive modulator of M1 differentiation in human monocytic cells [19]. Our current data, showing that TSP-1 overexpression induced the M1 phenotype in MH-S cells in vitro and ARDS in vivo, is consistent with these earlier publications.

4.2. TSP-2. The matricellular glycoprotein TSP-2 has been shown to regulate cell adhesion, migration, and proliferation. TSP-2 is also a strong inhibitor of angiogenesis [20]. In addition, inflammation associated with tissue injury has been identified as a powerful inducer of TSP-2 expression in murine tissues. TSP-2 has been shown to suppress the production of proinflammatory mediators, such as IFN- $\gamma$ and TNF- $\alpha$, and induce the depletion of tissue-residing T-cells [9]. Previous studies have also revealed that TSP-2 deficiency leads to an accelerated and increased influx of T-cells and monocytes/macrophages [20], and TSP-2-null mice display high inflammation, ECM deposition, and leakage of the blood-brain barrier (BBB) [21]. Consistently, we observed that TSP-2 overexpression in MH-S cells promoted the antiinflammatory M2 phenotype, which was accompanied by increased expression of the anti-inflammatory cytokine IL-10 and M2 markers Arg-1 and Fpr-3. ARDS is characterized by an excessive inflammatory response within the lungs and 


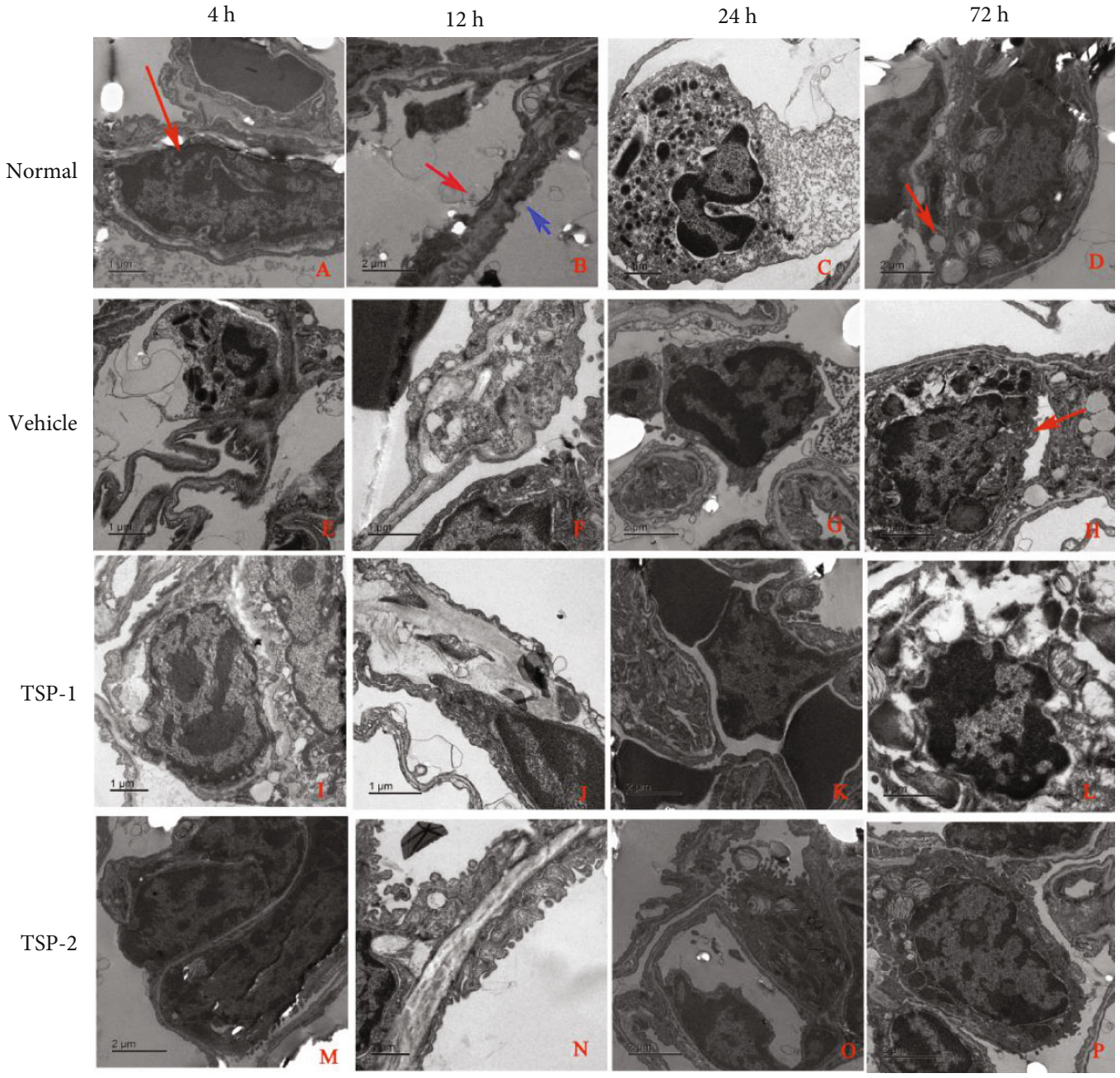

FIGURE 8: Ultrastructural morphology after TSP-2 overexpression in the ARDS mouse model. Mouse tissue sections from normal mice or mice treated with LPS for $4,12,24$, and $72 \mathrm{~h}$ and overexpressing either empty vector (vehicle group), TSP-1 (TSP-1 group), or TSP-2 (TSP-2 group) were analyzed for ultrastructural morphology using electron microscopy. (a-d) Ultrastructure of pulmonary capillaries under normal conditions. Arrow in (a) shows normal blood vessel epithelial cells (ECs), blue arrow in (b) shows the side of the bronchium, and the red arrow shows the side of the capillary at the air-blood barrier having a continuous thin basement membrane. (c) Macrophages within the blood vessel. Arrow in (d) shows alveolar epithelial cells (AECs) with clear lamellar bodies. (e-h) Electron micrographs in the vehicle group demonstrated disruption of the basement membrane in capillaries (e); thickened air-blood barrier (f); perivascular edema and inflammatory cell infiltration (g); and an apoptotic AEC with visible empty lamellar bodies, swollen cell organs, and contracted nuclei with condensed and fragmented nuclear chromatin (arrow) (h). (i-l) Ultrastructural morphology of EC (i), thickened air-blood barrier (j), perivascular edema (k), and apoptotic AEC (l) in the TSP-1 overexpression group, which was similar to the vehicle group. (m-p) Depiction of normal ultrastructural morphology of EC (m), AEC (p), air-blood barrier (n), and alleviated perivascular edema (o) in the TSP-2 overexpression group. The scale bars in (a, c, e, f, i, j, l, and n) are $1 \mu \mathrm{m}$; the scale bars in (b, d, g, h, k, $\mathrm{m}, \mathrm{o}$, and $\mathrm{p})$ are $2 \mu \mathrm{m}(n=5$ per group).

severely impaired gas exchange, resulting from alveolarcapillary barrier disruption and pulmonary edema [22]. One of the most important hallmarks of ARDS is high infiltration and accumulation of leukocytes in both interstitial and alveolar spaces. LPS-induced ARDS typically exhibits visible endothelial-epithelial damage and capillary-alveolar permeability, along with lung edema and tissue damage [22]. During our analysis of lung morphology and pathology of TSP-2-overexpressing ARDS mice, we observed that TSP-2 resulted in anti-inflammatory features, characterized by a reversal of LPS-induced endothelial-epithelial damage, reduced capillary-alveolar permeability, and inhibition of neutrophil migration and infiltration. These observations indicate the potential mechanism underlying improved endothelial cell proliferation and inhibition of adhesion/transmigration of inflammatory cells on blood vessel endothelial cells [20].

LPS treatment induces inflammatory factors and recruits neutrophils into the intravascular space across the endothelium and epithelium, as well as into the lungs and alveolar space, thereby promoting tissue damage [23]. The polarization of macrophages into the M1 phenotype has been linked to lung tissue damage in ARDS. Our results demonstrated that TSP-1 


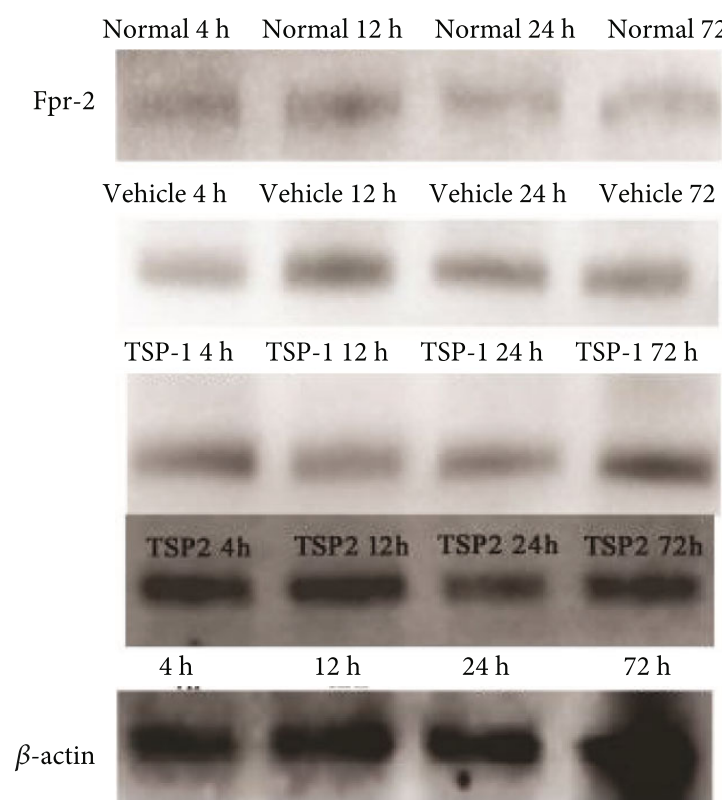

(a)
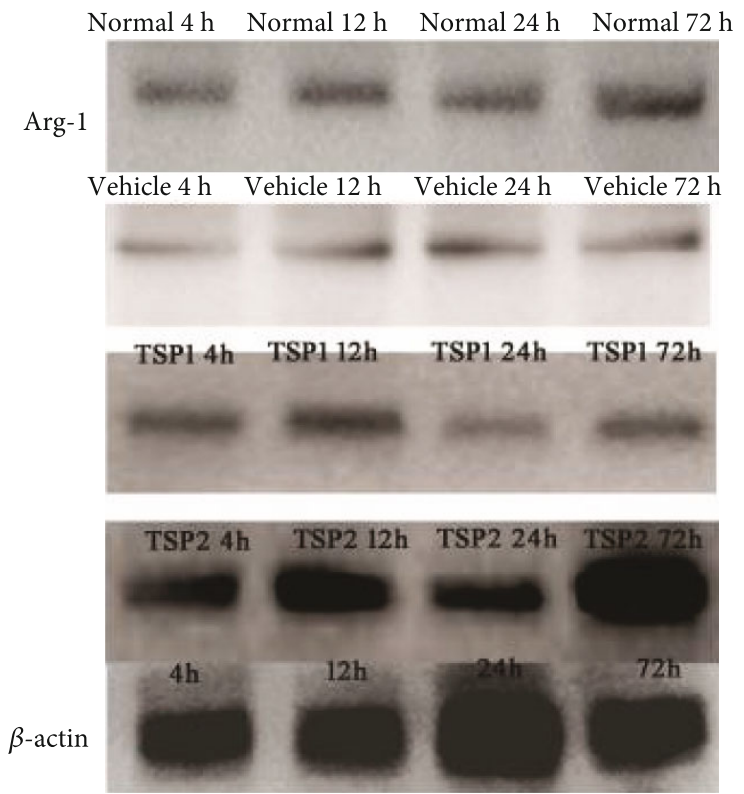

(c)

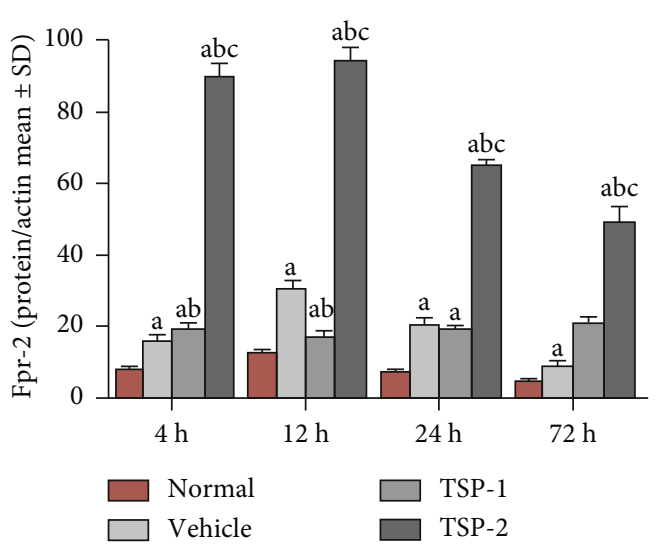

(b)

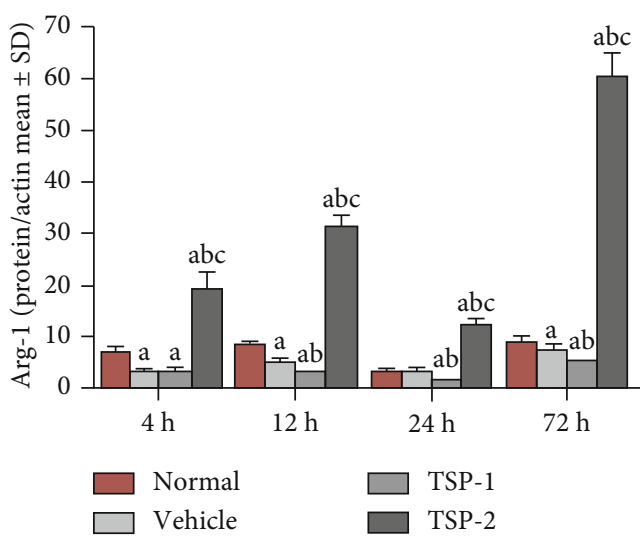

(d)

FIgURE 9: Continued. 

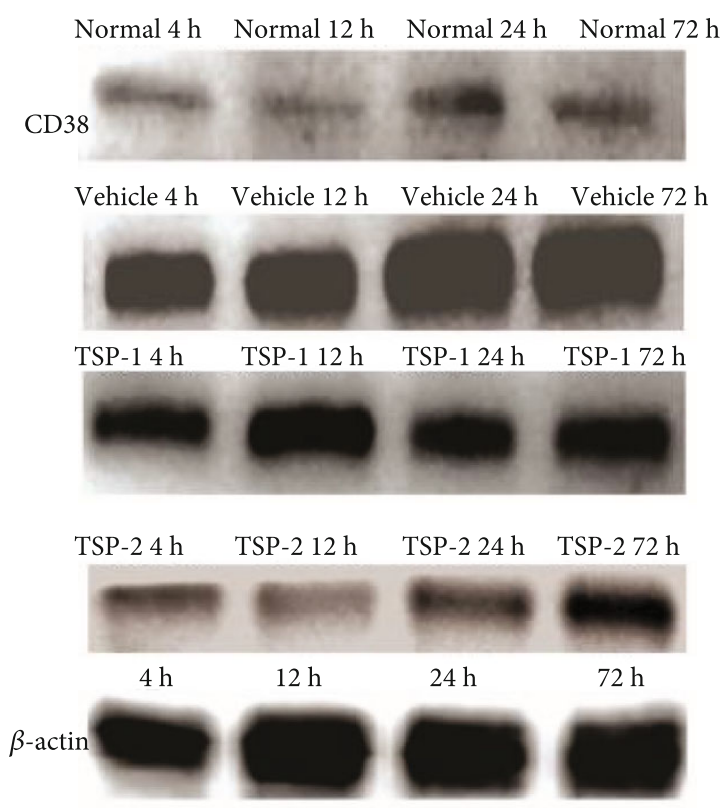

(e)

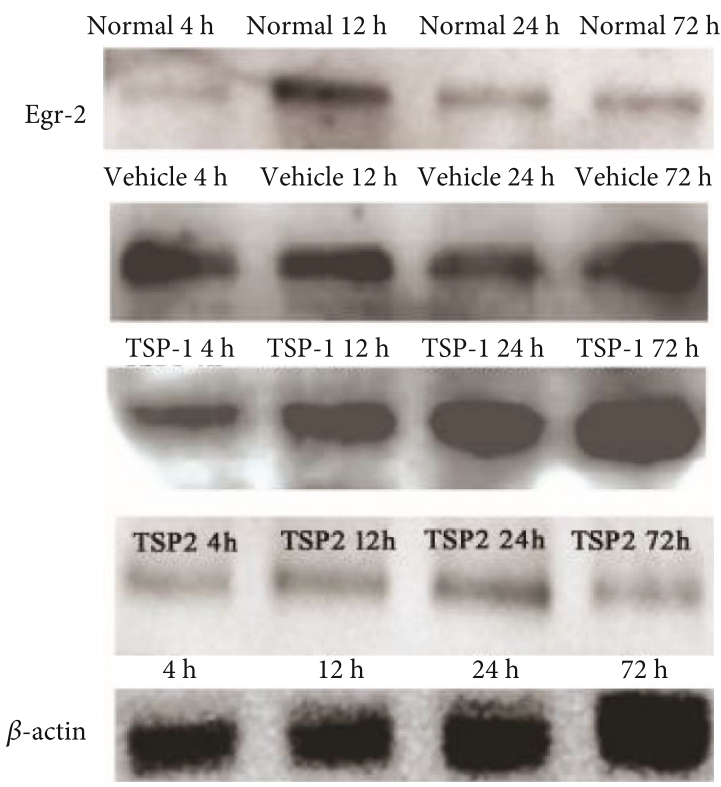

(g)

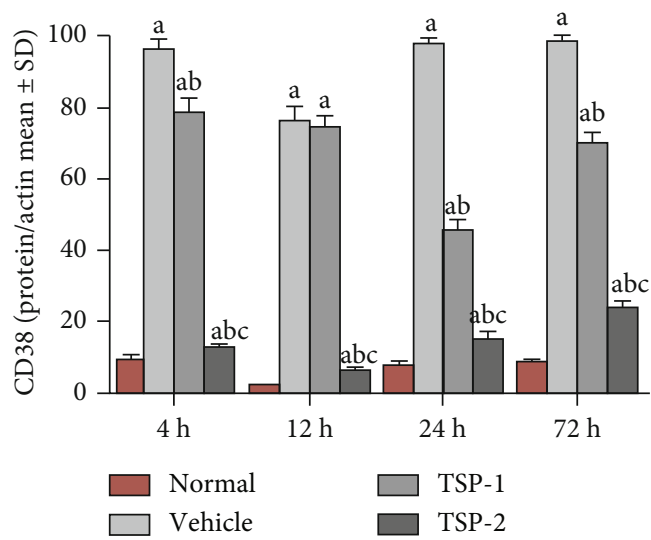

(f)

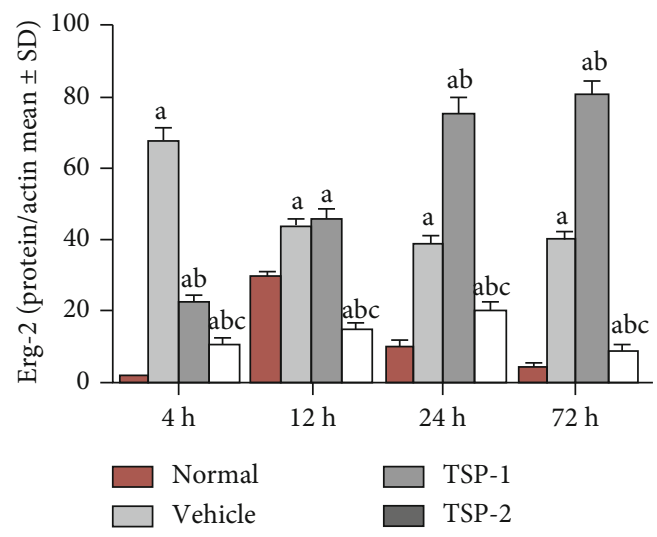

(h)

FIGURE 9: Effect of TSP overexpression on macrophage polarization in vivo. Mouse protein lysates from normal mice or mice treated with LPS for $4,12,24$, and $72 \mathrm{~h}$ and overexpressing either empty vector (vehicle group), TSP-1 (TSP-1 group), or TSP-2 (TSP-2 group) were analyzed for expression of M2 (Agr-1 and Egr-2) (a, c) and M1 (CD38 and Fpr-2) (e, g) macrophage markers using Western blot analysis. $\beta$-Actin expression served as the loading control. The bar graphs in (b, $\mathrm{d}, \mathrm{f}$, and $\mathrm{h}$ ) show the expression of these markers normalized to $\beta$-actin. The percentage of detected protein/actin is shown as mean \pm SD. $P$ value of $<0.05$ represents significant difference. ${ }^{\text {a }} P<0.05$ compared with normal control; ${ }^{\mathrm{b}} P<0.05$ compared with vehicle group; ${ }^{\mathrm{c}} P<0.05$ compared with TSP-1 transfected group ( $n=4$ per group).

overexpression promoted M1 macrophage polarization, which contributed to the deteriorating histopathology similar to LPS treatment of empty vector mice. Another independent study clearly demonstrated that M2 polarization significantly reduced lung inflammation and injury, reducing neutrophil influx into the lungs and augmenting apoptosis [24]. Consistent with this observation, TSP-2 overexpression in our study favored M2 macrophage polarization, and thus improved histo- pathologic changes in the lung tissue of TSP-2-overexpressing mice, as evidenced by decreases in lung injury and inflammation scores.

4.3. PI3K Signaling. TSP-2 is associated with the PI3K/Akt signaling pathway [25]. A previous study demonstrated that Akt, p-Akt, and PI3K expression were drastically reduced following TSP-2 silencing. Moreover, these cells exhibited 

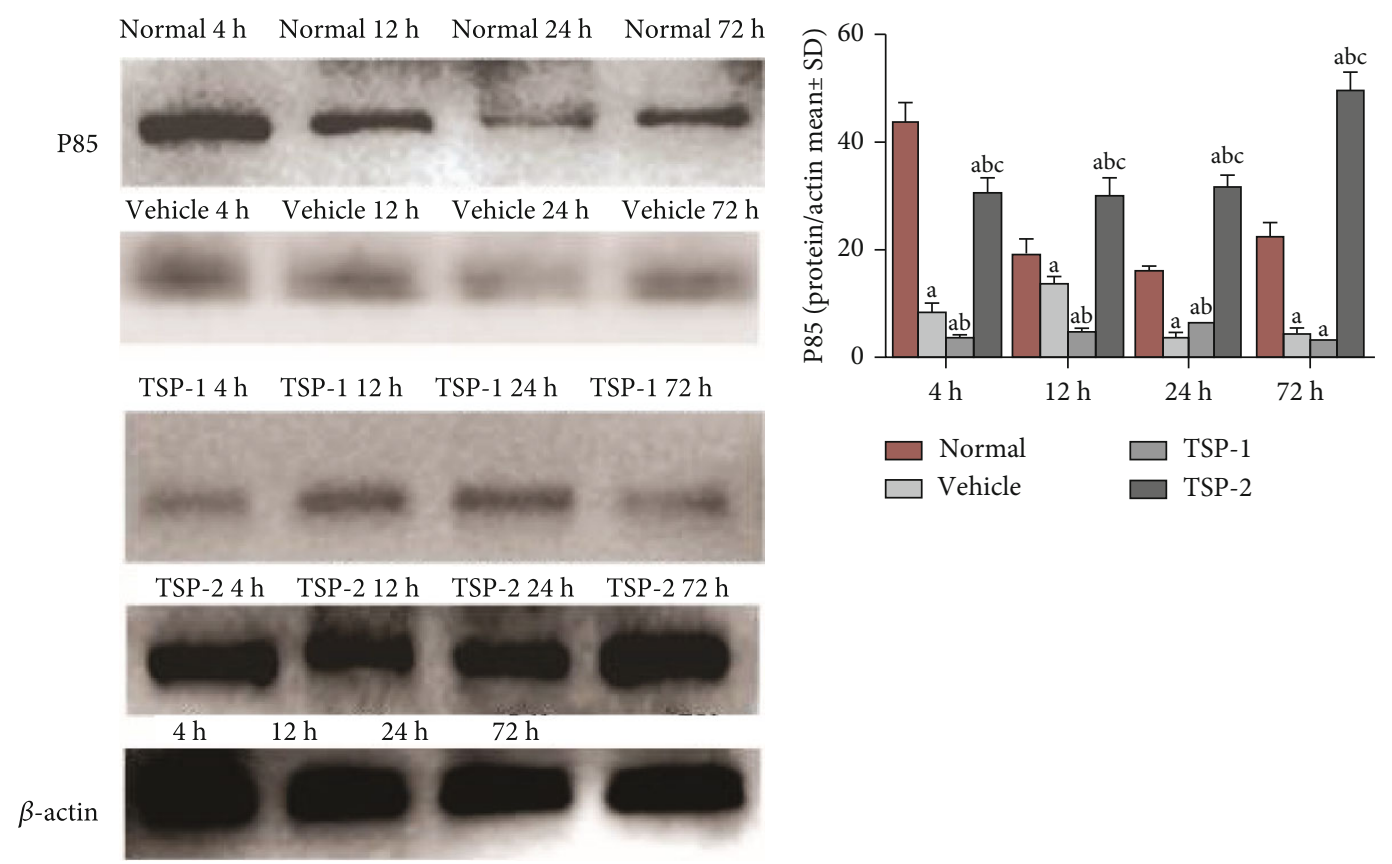

(a)

(b)

Normal 4 h Normal 12 h Normal 24 h Normal 72 h

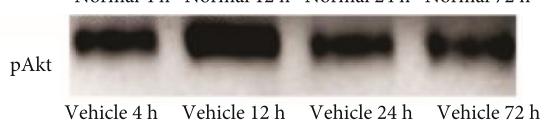

Vehicle $4 \mathrm{~h} \quad$ Vehicle $12 \mathrm{~h} \quad$ Vehicle $24 \mathrm{~h} \quad$ Vehicle $72 \mathrm{~h}$

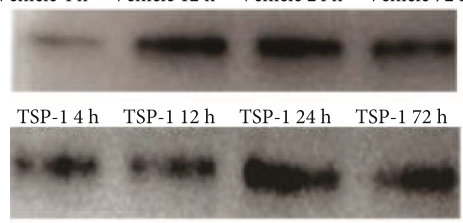

TSP2 4h TSP2 12h TSP2 24h TSP2 72h
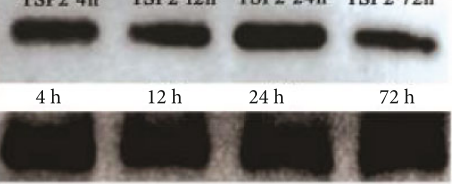

$\beta$-actin

Vehicle $4 \mathrm{~h} \quad$ Vehicle $12 \mathrm{~h} \quad$ Vehicle $24 \mathrm{~h} \quad$ Vehicle $72 \mathrm{~h}$

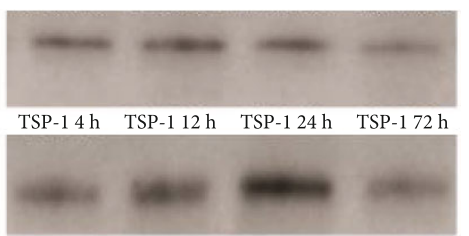

TSP2 4h TSP2 12h TSP2 24h TSP2 72h

$4 \mathrm{~h} \quad 12 \mathrm{~h} \quad 24 \mathrm{~h} \quad 72 \mathrm{~h}$

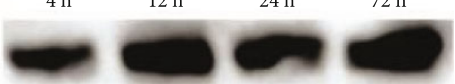

c)

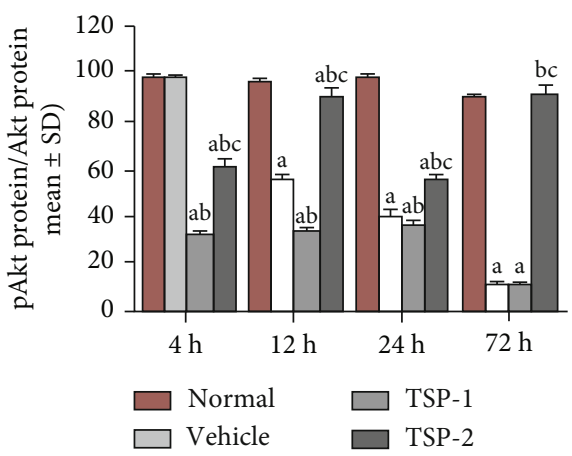

(d)

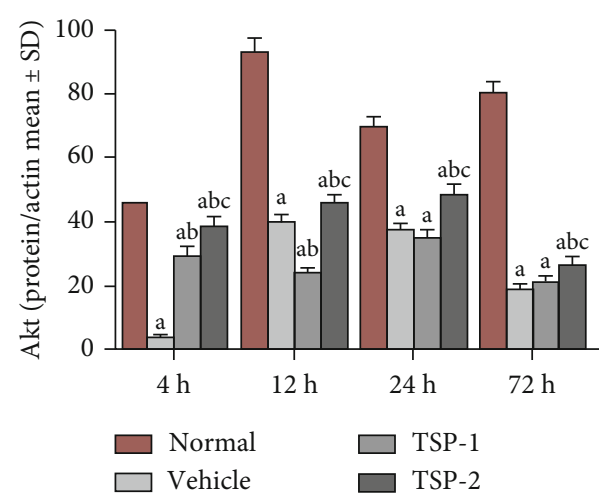

(e)

Figure 10: Continued. 


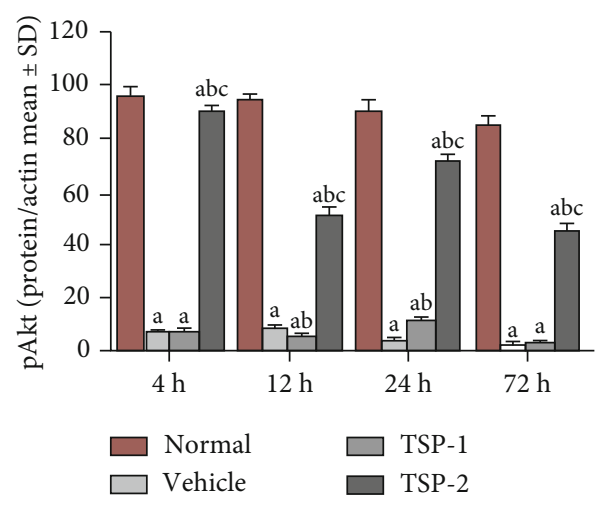

(f)

FIGURE 10: Effect of TSP overexpression on PI3K signaling in vivo. Mouse protein lysates from normal mice or mice treated with LPS for 4, 12, 24, and $72 \mathrm{~h}$ and overexpressing either empty vector (vehicle group), TSP-1 (TSP-1 group), or TSP-2 (TSP-2 group) were analyzed for p85 (a), $\mathrm{p}$-Akt, and total Akt (c) expression using Western blot analysis. $\beta$-Actin expression served as the loading control. (b, e, and $\mathrm{f}$ ) Bar graphs are shown after normalizing the expression of $\mathrm{p} 85$, total Akt, and $\mathrm{p}$-Akt to $\beta$-actin. (d) Bar graph for $\mathrm{p}$-Akt expression is shown after normalizing to total Akt expression. The percentage of detected protein/actin is shown as mean \pm SD. $P$ value of $<0.05$ represents significant difference. ${ }^{\mathrm{a}} P<0.05$ compared with normal control; ${ }^{\mathrm{b}} P<0.05$ compared with vehicle group; ${ }^{\mathrm{c}} P<0.05$ compared with the TSP-1 transfected group $(n=4$ per group).

enhanced apoptosis and reduced proliferation [25]. In human pulmonary microvascular endothelial cells, it has been observed that LPS-mediated dysregulation of barrier function involved PI3K/Akt signaling in a dose-dependent manner [26]. In addition, levels of p-Akt were dramatically reduced in the ARDS mouse model, and treatment with the PI3K inhibitor, wortmannin, further enhanced lung injury [27]. We observed consistent changes in PI3K/Akt signaling both after TSP-2 overexpression in MH-S cells in vitro and in the macrophage in vivo model. Inhibition of the PI3K/Akt pathway by LY294002 resulted in a significant increase in CD68 expression in mice transfected with TSP-2, as well as further exacerbated CD68 expression in mice transfected with TSP-1, thereby confirming the relationship between $\mathrm{PI} 3 \mathrm{~K} / \mathrm{Akt}$ pathway activation and inflammation. This suggests that the effects of TSP-2 on suppressing inflammation were at least partly achieved through activation of the PI3K/Akt pathway. Consistent with our data, previous studies have also reported that genipin pretreatment significantly activated PI3K/Akt signaling and alleviated LPSinduced inflammation. However, LY294002 specifically inhibited the protective effects of genipin, including the effects on apoptosis, endoplasmic reticulum stress (ERS), and inflammation in an LPS-induced acute lung injury (ALI) model [28]. Another study showed that fibroblast growth factor-2 (FGF-2) effectively reduced LPS-induced inflammation, oxidative stress, and apoptosis in alveolar epithelial cells and increased activation of the PI3K/Akt signaling pathway. LY294002 treatment also alleviated the protective effect of FGF-2 in the lung tissue [29]. Therefore, it is reasonable to assume that activating the PI3K/Akt signaling pathway is beneficial for protecting the lung tissue. In addition, we showed that TSP-2 overexpression upregulated p-Akt, Akt, and p85 (a regulatory subunit of PI3K) following LPS treatment, and thus enhanced cell viability and inhibited apoptosis, which was consistent with previously published studies [30, 31].
However, we observed an opposite effect of TSP-1 on PI3K signaling in our in vitro and in vivo assays. Our in vitro study showed that TSP-1 overexpression restored p85 and Akt levels within $0.5 \mathrm{~h}$ of LPS treatment, while our in vivo data indicated that TSP-1 overexpression reduced p85, p-Akt, and total Akt protein levels compared to the normal control after 4, 12, 24, and $72 \mathrm{~h}$ of LPS injection. These differences can be attributed to the different time points at which expression levels were assessed. The early time point effects of TSP-1 overexpression on PI3K signaling can be due to its effect on severe pulmonary vascular remodeling, peripheral vascular rarefaction, and fibrosis, which can subsequently limit lung function at later stages. Consistent with this data, previous studies have also shown that $A k t 1^{-l-}$ mice were protected from chronic hypoxia-induced pulmonary vascular tissue remodeling and fibrosis, and TSP-1 can work as a matricellular protein in hypoxia-induced pulmonary remodeling [32]. In the same study, lungs from $T S P 1^{-/-}$mice were also analyzed to determine resistance to pulmonary fibrosis induced by adMyrAkt1 overexpression.

4.4. TSP Overexpression as Gene Therapy. The potential of using TSPs as gene therapy has been explored for quite some time. In 2002, one study was the first to suggest that TSP-2 gene therapy can act as an antiangiogenic tumor therapy. Herein, a tissue-engineered implant system was created with an ability to continuously produce TSP-2 in vivo, with the intent to potently inhibit endogenous tumor growth and angiogenesis. In this system, fibroblasts were retrovirally transduced to overexpress TSP-2 and were seeded onto biodegradable polymer scaffolds. This bio-implant-generated TSP-2 potently inhibited tumor growth and angiogenesis of human squamous cell carcinomas, malignant melanomas, and Lewis lung carcinomas [33]. Similarly, other studies have also demonstrated the potential of TSP-2 gene therapy to ameliorate experimental glomerulonephritis via inhibiting cell proliferation, inflammation, and TGF- $\beta$ activation. In one 


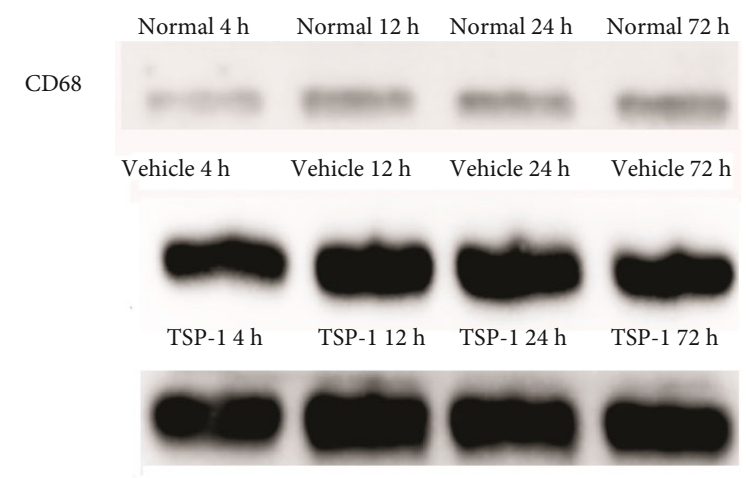

TSP-1+LY 4 h TSP-1+LY 12 h TSP-1+LY 24 h TSP-1+LY 72 h

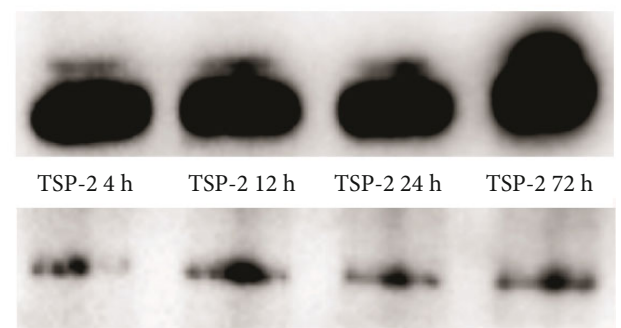

TSP-2+LY 4 h TSP-2+LY 12 h TSP-2+LY 24 h TSP-2+LY 72 h
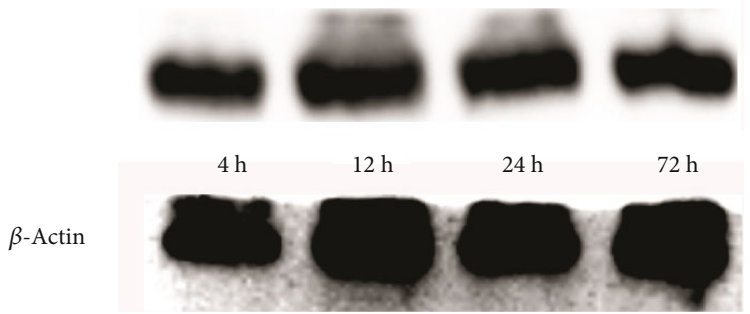

(a)

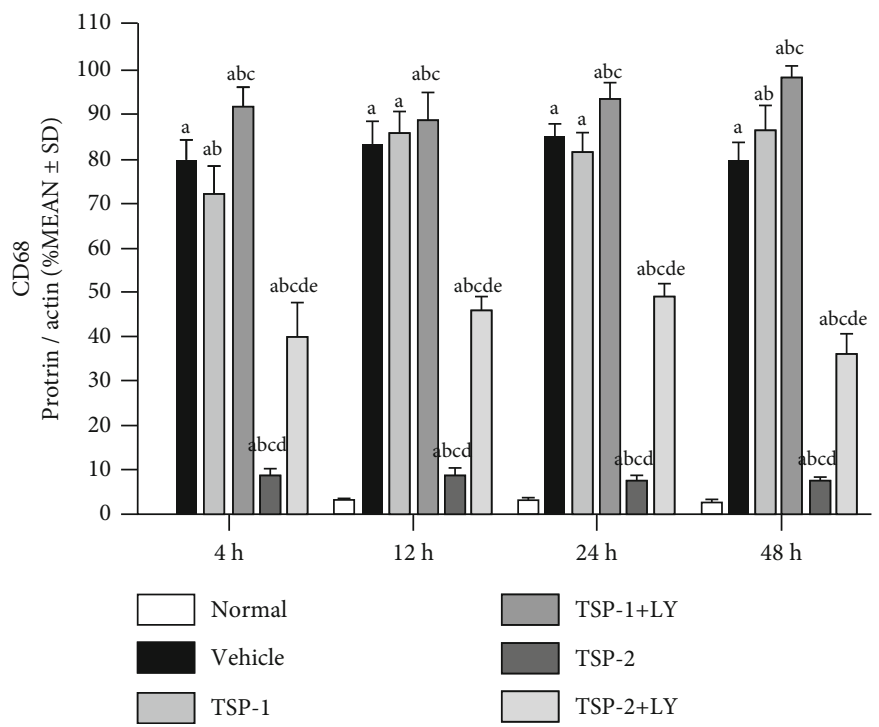

(b)

FIGURE 11: Effect of PI3K/Akt signaling inhibitor LY294002 on lung inflammation in vivo. The expression of CD68, a macrophage marker, in protein lysates of mice treated with LPS for $4,12,24$, and $72 \mathrm{~h}$ and overexpressing either empty vector (vehicle group), TSP-1 (TSP-1 group), or TSP-2 (TSP-2 group), as well as the TSP-1/TSP-2+LY204002 groups, are shown using Western blot analysis. $\beta$-Actin expression served as the loading control. The percentage of detected protein/actin is shown as mean \pm SD. $P$ value of $<0.05$ represents significant difference. ${ }^{\mathrm{a}} P<0.05$ compared with normal control; ${ }^{\mathrm{b}} \mathrm{P}<0.05$ compared with vehicle control; ${ }^{\mathrm{c}} P<0.05$ compared with the TSP-1 group; ${ }^{\mathrm{d}} P<0.05$ compared with the TSP-1 transfected+LY294200 group; ${ }^{\mathrm{e}} \mathrm{P}<0.05$ compared with the TSP- 2 transfected group ( $n=4$ per group). 
such study, TSP-2 overexpression inhibited both glomerular endothelial and mesangial cell proliferation, resulting in reduced glomerular cell number and glomerular tuft area [34]. Importantly, the inflammatory response, as monitored by $\mathrm{T}$-cell and antigen-presenting cell infiltration, was also significantly reduced after TSP-2 overexpression [34]. In chronic allograft nephropathy, long-term TSP-2 gene therapy led to the inhibition of TGF- $\beta$ activation, inflammation, and angiogenesis [35]. Together, these studies provide a strong foundation for translating TSP gene therapy to the clinic.

\section{Conclusions}

In conclusion, macrophages, which can be either inflammatory or anti-inflammatory, can overexpress various thrombospondins that can induce macrophage polarization. Subsequently, these polarized macrophages play different roles in pulmonary inflammation. Our study specifically demonstrated that TSP-1 overexpression promoted M1 macrophage polarization and enhanced LPS-induced inflammation, while TSP-2 overexpression effectively attenuated LPS-induced ARDS by promoting M2 macrophage polarization. These findings lead us to conclude that TSP-2 could be developed as a potential therapeutic target against LPS-induced ARDS.

\section{Data Availability}

The datasets generated and analyzed during the current study are available from the corresponding authors on reasonable request.

\section{Conflicts of Interest}

The authors declare that they have no conflict of interest.

\section{Authors' Contributions}

Jiang Yuan and Shu Han contributed equally to this work.

\section{Acknowledgments}

This work was supported by the Natural Science Foundation of China (project Nos. 81801552 and 81971069) and the Zhejiang Provincial Natural Science Foundation of China (project No. LY19H1500003).

\section{Supplementary Materials}

Supplementary 1. Figure SP1 Expression analyses of TSP-1 and TSP-2 overexpression in vivo. The mouse tissue sections from normal mice or mice treated with LPS for 4, 12, 24, and $72 \mathrm{~h}$ and overexpressing either empty vector (vehicle group), TSP-1 (TSP-1 group), or TSP-2 (TSP-2 group) were analyzed for TSP-1 (A) and TSP-2 (C) protein expression using Western blot analysis. $\beta$-Actin expression served as a loading control. (B, D) represented TSP-1 and TSP-2 expressions after normalization to $\beta$-actin expression in all four groups. The expression percentage of detected proteins/actin is shown as mean $\pm \mathrm{SD}$. $P$ value of $<0.05$ represents significant difference. ${ }^{\mathrm{a}} P<0.05$ compared with normal control; ${ }^{\mathrm{b}} \mathrm{P}<0.05$ compared with vehicle group; ${ }^{c} P<0.05$ compared with TSP-1 transfected group ( $n=4$ per group).

Supplementary 2. Figure SP2. TSP-2 effects on inflammatory cell accumulation in BALF. (A-M) Analyses of inflammatory cells using Wright-Giemsa staining in BALF collected from mice treated with LPS for 4, 12, 24, and $72 \mathrm{~h}$ and overexpressing either empty vector (vehicle group), TSP-1 (TSP-1 group), or TSP-2 (TSP-2 group). (M) Wright-Giemsa staining in BALF from normal mice. Scale bar $=100 \mu \mathrm{m}$. (N) Five sections were randomly selected, and images were photographed under 400x magnification. Leukocyte numbers were counted from three fields per section. The number of leukocytes in each group is shown as mean \pm SD. $P$ value of $<0.05$ represents significant difference; ${ }^{*} P<0.05$ vs. normal group; ${ }^{\#} P<0.05$ vs. vehicle group ( $n=3$ per group).

Supplementary 3. Figure SP3. Effect of TSP-2 on active caspase-3 in vivo. (A-L) Caspase-3 staining (red color) in lung tissue sections of mice treated with LPS for 4, 12, 24, and $72 \mathrm{~h}$ and overexpressing either empty vector (vehicle group), TSP-1 (TSP-1 group), or TSP-2 (TSP-2 group). (M) Caspase-3 staining in lung sections from normal mice. Scale bar $=100 \mu \mathrm{m} .(n=5$ per group $)$.

\section{References}

[1] M. A. Matthay, L. B. Ware, and G. A. Zimmerman, "The acute respiratory distress syndrome," The Journal of Clinical Investigation, vol. 122, no. 8, pp. 2731-2740, 2012.

[2] S. Herold, K. Mayer, and J. Lohmeyer, "Acute lung injury: how macrophages orchestrate resolution of inflammation and tissue repair," Frontiers in Immunology, vol. 2, p. 65, 2011.

[3] K. Hiraiwa and S. F. van Eeden, "Contribution of lung macrophages to the inflammatory responses induced by exposure to air pollutants," Mediators of Inflammation, vol. 2013, Article ID 619523, 10 pages, 2013.

[4] J. Wei, G. Chen, X. Shi et al., "Nrf2 activation protects against intratracheal LPS induced mouse/murine acute respiratory distress syndrome by regulating macrophage polarization," Biochemical and Biophysical Research Communications, vol. 500, no. 3, pp. 790-796, 2018.

[5] A. P. Papageorgiou, M. Swinnen, D. Vanhoutte et al., "Thrombospondin-2 prevents cardiac injury and dysfunction in viral myocarditis through the activation of regulatory T-cells," Cardiovascular Research, vol. 94, no. 1, pp. 115-124, 2012.

[6] Z. Lopez-Dee, K. Pidcock, and L. S. Gutierrez, "Thrombospondin-1: multiple paths to inflammation," Mediators of Inflammation, vol. 2011, Article ID 296069, 10 pages, 2011.

[7] T. Xing, Y. Wang, W. J. Ding et al., “Thrombospondin-1 production regulates the inflammatory cytokine secretion in THP-1 cells through NF- $\kappa$ B signaling pathway," Inflammation, vol. 40, no. 5, pp. 1606-1621, 2017.

[8] E. V. Stein, T. W. Miller, K. Ivins-O’Keefe, S. Kaur, and D. D. Roberts, "Secreted thrombospondin-1 regulates macrophage interleukin- $1 \beta$ production and activation through CD47," Scientific Reports, vol. 6, no. 1, article 19684, 2016.

[9] Y. W. Park, Y. M. Kang, J. Butterfield, M. Detmar, J. J. Goronzy, and C. M. Weyand, "Thrombospondin 2 functions as an endogenous regulator of angiogenesis and inflammation 
in rheumatoid arthritis," The American Journal of Pathology, vol. 165, no. 6, pp. 2087-2098, 2004.

[10] Q. H. Liu and L. S. Ma, "Knockdown of thrombospondin 2 inhibits metastasis through modulation of PI3K signaling pathway in uveal melanoma cell line M23," European Review for Medical and Pharmacological Sciences, vol. 22, no. 19, pp. 6230-6238, 2018.

[11] K. Matsunaga, T. W. Klein, H. Friedman, and Y. Yamamoto, "Alveolar macrophage cell line MH-S is valuable as an in vitro model for Legionella pneumophila infection," American Journal of Respiratory Cell and Molecular Biology, vol. 24, no. 3, pp. 326-331, 2001.

[12] S. Han, F. Zhang, Z. Hu et al., "Dose-dependent antiinflammatory and neuroprotective effects of an $\alpha v \beta 3$ integrin-binding peptide," Mediators of Inflammation, vol. 2013, Article ID 268486, 24 pages, 2013.

[13] B. Wang, K. W. Tian, F. Zhang, H. Jiang, and S. Han, “Angiopoietin-1 and C16 peptide attenuate vascular and inflammatory responses in experimental allergic encephalomyelitis," CNS \& Neurological Disorders Drug Targets, vol. 15, no. 4, pp. 496-513, 2016.

[14] Q. Ding, G. Q. Liu, Y. Y. Zeng et al., "Role of IL-17 in LPSinduced acute lung injury: an in vivo study," Oncotarget, vol. 8, no. 55, pp. 93704-93711, 2017.

[15] D. Qi, D. Wang, C. Zhang et al., "Vaspin protects against LPS-induced ARDS by inhibiting inflammation, apoptosis and reactive oxygen species generation in pulmonary endothelial cells via the Akt/GSK-3 $\beta$ pathway," International Journal of Molecular Medicine, vol. 40, no. 6, pp. 1803-1817, 2017.

[16] Z. Liu, S. Morgan, J. Ren et al., “Thrombospondin-1 (TSP1) contributes to the development of vascular inflammation by regulating monocytic cell motility in mouse models of abdominal aortic aneurysm," Circulation Research, vol. 117, no. 2, pp. 129-141, 2015.

[17] E. Sick, A. Jeanne, C. Schneider, S. Dedieu, K. Takeda, and L. Martiny, "CD47 update: a multifaceted actor in the tumour microenvironment of potential therapeutic interest," British Journal of Pharmacology, vol. 167, no. 7, pp. 1415-1430, 2012.

[18] Y. Li, X. Qi, X. Tong, and S. Wang, "Thrombospondin 1 activates the macrophage Toll-like receptor 4 pathway," Cellular \& Molecular Immunology, vol. 10, no. 6, pp. 506-512, 2013.

[19] G. Martin-Manso, S. Galli, L. A. Ridnour, M. Tsokos, D. A. Wink, and D. D. Roberts, "Thrombospondin 1 promotes tumor macrophage recruitment and enhances tumor cell cytotoxicity of differentiated U937 cells," Cancer Research, vol. 68, no. 17, pp. 7090-7099, 2008.

[20] C. Daniel, K. Amann, B. Hohenstein, P. Bornstein, and C. Hugo, "Thrombospondin 2 functions as an endogenous regulator of angiogenesis and inflammation in experimental glomerulonephritis in mice," Journal of the American Society of Nephrology, vol. 18, no. 3, pp. 788-798, 2007.

[21] W. Tian, A. Sawyer, F. B. Kocaoglu, and T. R. Kyriakides, "Astrocyte-derived thrombospondin-2 is critical for the repair of the blood-brain barrier," The American Journal of Pathology, vol. 179, no. 2, pp. 860-868, 2011.

[22] X. Huang, H. Xiu, S. Zhang, and G. Zhang, "The role of macrophages in the pathogenesis of ALI/ARDS," Mediators of Inflammation, vol. 2018, Article ID 1264913, 8 pages, 2018.

[23] Y. Li, J. Huang, N. M. Foley et al., "B7H3 ameliorates LPSinduced acute lung injury via attenuation of neutrophil migra- tion and infiltration," Scientific Reports, vol. 6, no. 1, article 31284, 2016.

[24] G. W. Tu, Y. Shi, Y. J. Zheng et al., "Glucocorticoid attenuates acute lung injury through induction of type 2 macrophage," Journal of Translational Medicine, vol. 15, no. 1, p. 181, 2017.

[25] R. Ao, L. Guan, Y. Wang, and J. N. Wang, "Silencing of COL1A2, COL6A3, and THBS2 inhibits gastric cancer cell proliferation, migration, and invasion while promoting apoptosis through the PI3k-Akt signaling pathway," Journal of Cellular Biochemistry, vol. 119, no. 6, pp. 4420-4434, 2018.

[26] X. Zheng, W. Zhang, and X. Hu, "Different concentrations of lipopolysaccharide regulate barrier function through the PI3K/Akt signalling pathway in human pulmonary microvascular endothelial cells," Scientific Reports, vol. 8, no. 1, p. 9963, 2018.

[27] S. Ji and L. Wang, “ $\mu$-Opioid receptor signalling via PI3K/Akt pathway ameliorates lipopolysaccharide-induced acute respiratory distress syndrome," Experimental Physiology, vol. 104, no. 10, pp. 1555-1561, 2019.

[28] X. Luo, B. Lin, Y. Gao et al., "Genipin attenuates mitochondrial-dependent apoptosis, endoplasmic reticulum stress, and inflammation via the PI3K/AKT pathway in acute lung injury," International Immunopharmacology, vol. 76, article 105842, 2019.

[29] Q. Y. Tang, J. X. Wei, S. F. Xue, G. H. Liu, and L. X. Fu, "Fibrogrowth factor-2 protects against acute lung injury by activating the PI3K/Akt signaling pathway," Journal of Biological Regulators and Homeostatic Agents, vol. 34, no. 5, pp. 1679-1688, 2020.

[30] Y. Jia, S. J. Mo, Q. Q. Feng et al., "EPO-dependent activation of $\mathrm{PI}$ K/Akt/FoxO3a signalling mediates neuroprotection in in vitro and in vivo models of Parkinson's disease," Journal of Molecular Neuroscience, vol. 53, no. 1, pp. 117-124, 2014.

[31] J. Wang, J. Liu, Y. Wang et al., "High glucose induces alternative activation of macrophages via PI3K/Akt signaling pathway," Journal of Receptor and Signal Transduction Research, vol. 37, no. 4, pp. 409-415, 2017.

[32] M. Abdalla, H. Sabbineni, R. Prakash, A. Ergul, S. C. Fagan, and P. R. Somanath, "The Akt inhibitor, triciribine, ameliorates chronic hypoxia-induced vascular pruning and TGF $\beta$ induced pulmonary fibrosis," British Journal of Pharmacology, vol. 172, no. 16, pp. 4173-4188, 2015.

[33] M. Streit, A. E. Stephen, T. Hawighorst et al., "Systemic inhibition of tumor growth and angiogenesis by thrombospondin-2 using cell-based antiangiogenic gene therapy," Cancer Research, vol. 62, no. 7, pp. 2004-2012, 2002.

[34] C. Daniel, A. Wagner, B. Hohenstein, and C. Hugo, "Thrombospondin-2 therapy ameliorates experimental glomerulonephritis via inhibition of cell proliferation, inflammation, and TGF-beta activation," American Journal of Physiology. Renal Physiology, vol. 297, no. 5, pp. F1299-F1309, 2009.

[35] C. Daniel, R. Vogelbacher, A. Stief, C. Grigo, and C. Hugo, "Long-term gene therapy with thrombospondin 2 inhibits TGF- $\beta$ activation, inflammation and angiogenesis in chronic allograft nephropathy," PLoS One, vol. 8, no. 12, article e83846, 2013. 